\title{
Radiation shielding for the Main Injector collimation system*
}

\author{
Igor Rakhno \\ Fermilab, P.O. Box 500, Batavia, IL 60510
}

December 7, 2007

\begin{abstract}
The results of Monte Carlo radiation shielding studies performed with the MARS 15 code for the Main Injector collimation system are presented and discussed.
\end{abstract}

\section{Introduction}

A Proton Plan was developed recently at Fermilab for the benefit of the existing neutrino programs as well as to increase anti-proton production for the Tevatron programs [1]. As a part of the plan, the intensity of proton beams in the Main Injector (MI) should be increased by means of slip-stacking injection. In order to localize beam loss associated with the injection, a collimation system was designed [2] that satisfies all the radiation and engineering constraints. The system itself comprises a primary collimator and four secondary ones to which various masks are added. It is assumed that beam loss at the slip-stacking injection is equal to $5 \%$ of total intensity which is $5.5 \times 10^{13}$ protons per pulse [2]. As far as pulse separation is 2.2 seconds, one has $\left(5.5 \times 10^{13} / 2.2\right) \times 0.05=1.25 \times 10^{12}$ protons interacting per second with the primary collimator.

In the paper the geometry model of the corresponding MI region and beam loss model are described. The model of the region was built by means of the MAD-MARS Beam Line Builder (MMBLB) [3] using results of the collimation studies [2]. The results of radiation shielding calculations performed with the MARS15 code [4] are presented.

\section{Geometry Model of the Region}

The geometry model developed for the MI collimation region is shown in Figs. 1 thru 5. The model extends from Q230 quadrupole up to Q310, i.e. for about 200 meters, and

*Work supported by the Universities Research Association, Inc., under contract DE-AC02-76CH03000 with the U. S. Department of Energy. 


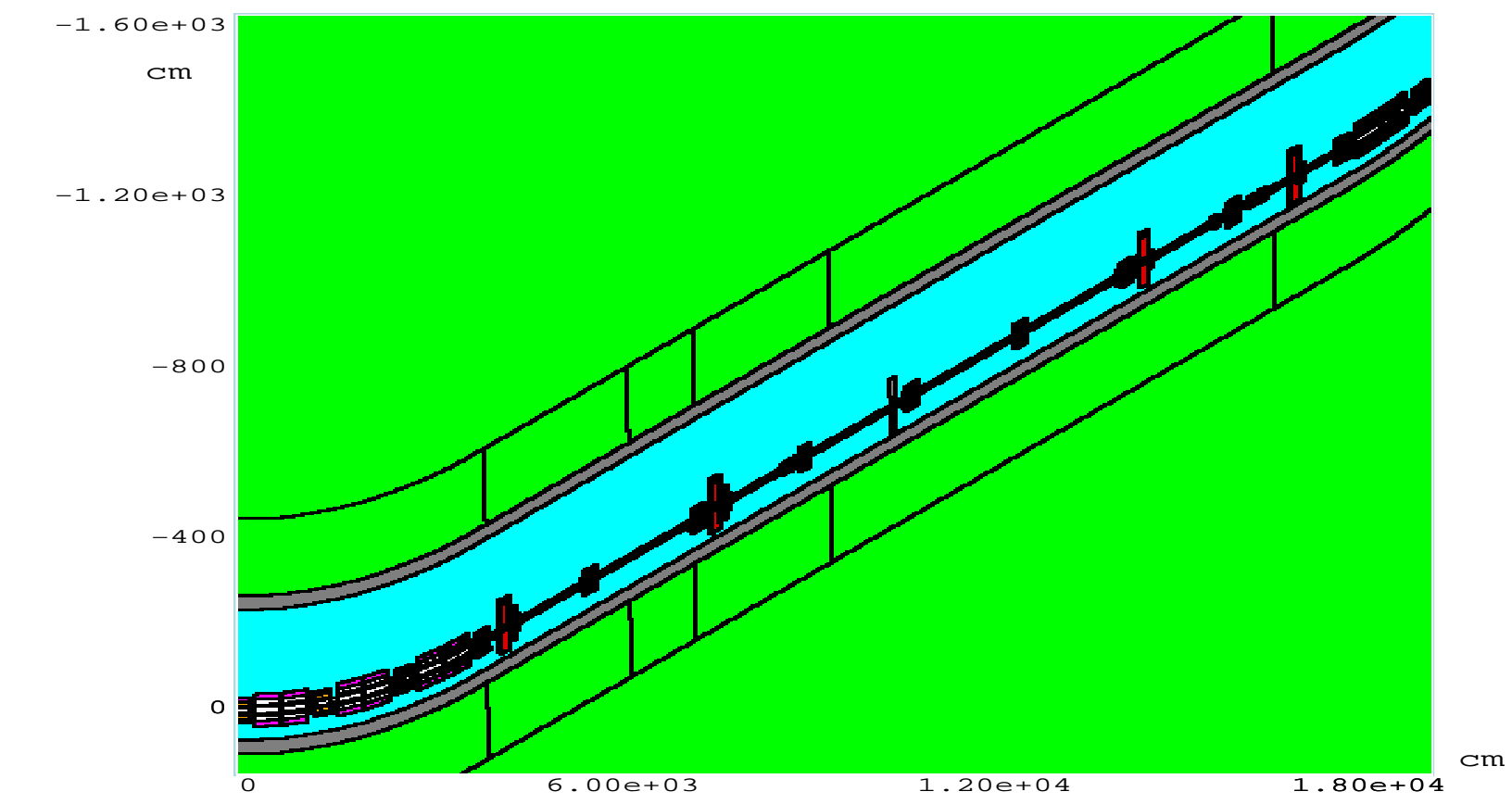

$\underset{Y}{\longrightarrow}$

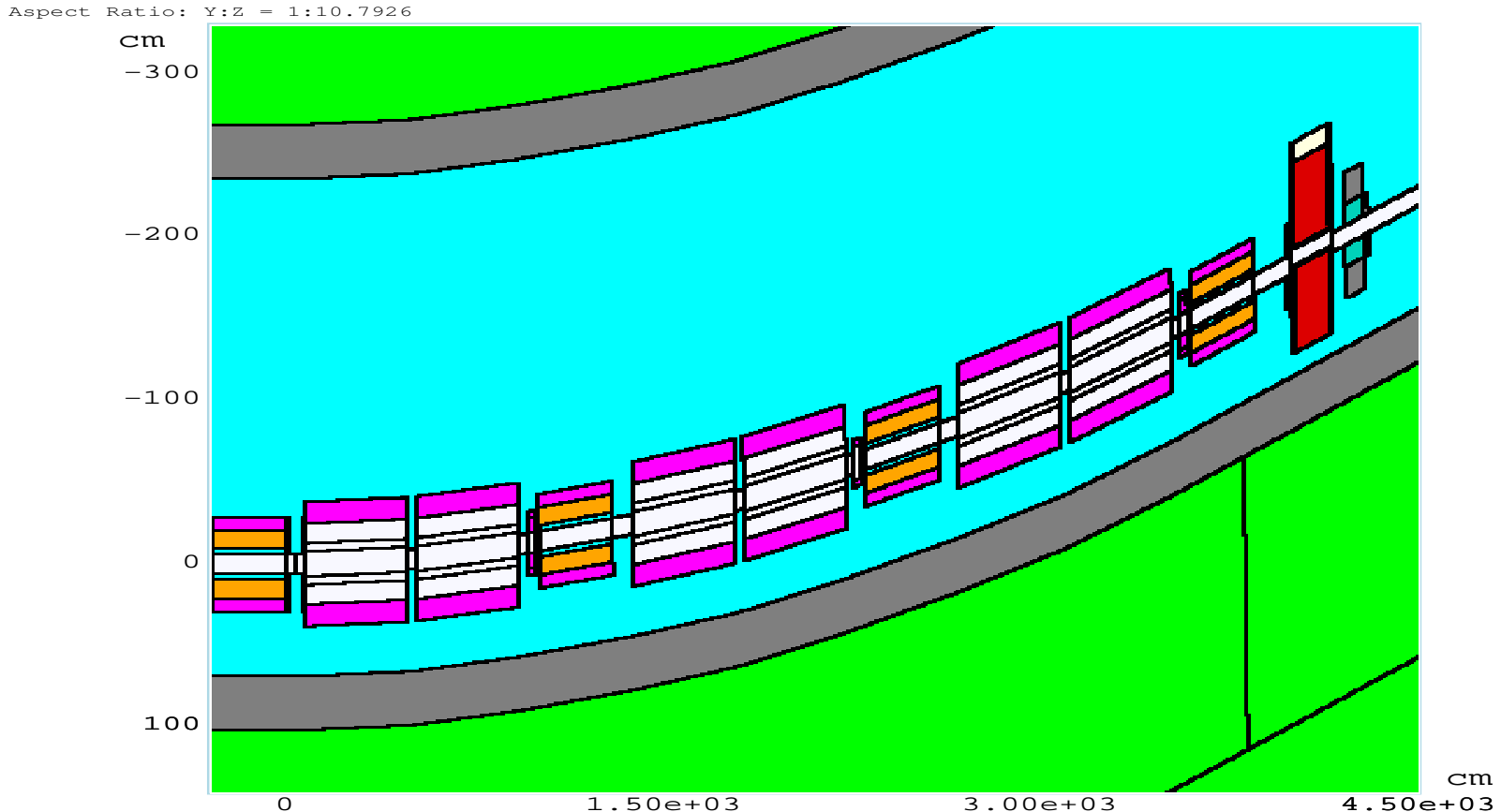

$\downarrow_{Y}^{\text {z }}$

Figure 1: A plan view of the MARS15 model of the entire collimation region from Q230 up to Q310 (top) and a fragment of the model from Q230 up to the first secondary collimator S1 (bottom). The lines thru the dirt outside of the tunnel are used to define the so-called 99-\% volume (see Sec. 4.1). 

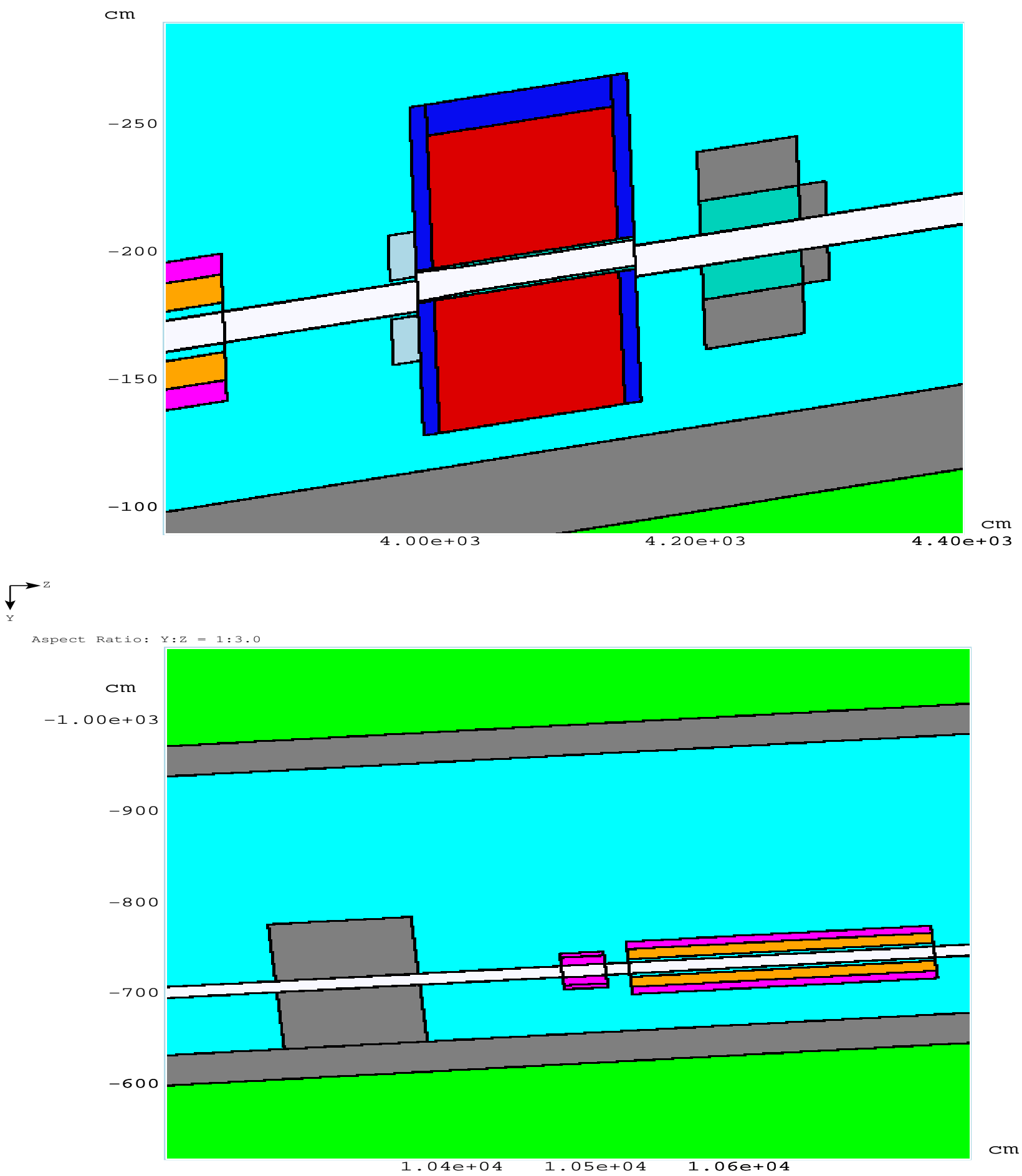

$\underset{Y}{\downarrow}$

Aspect Ratio: $\mathrm{Y}: \mathrm{z}=1: 1.0$

Figure 2: A plan view of the MARS15 model of the first secondary collimator S1 with its upstream and downstream masks (top) and a fragment with the Q305 quadrupole with concrete wall upstream (bottom). 

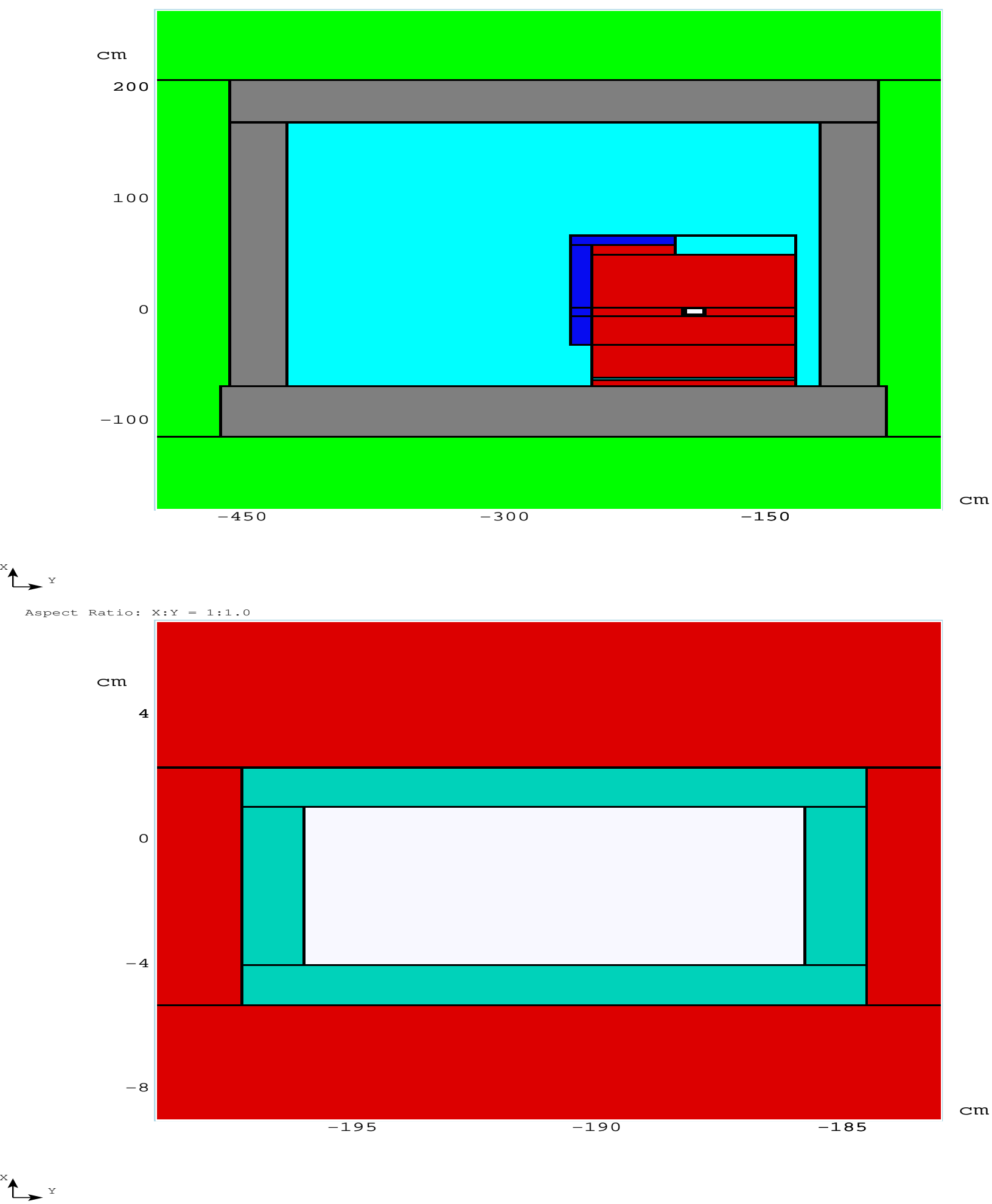

Aspect Ratio: $\mathrm{X}: \mathrm{Y}=1: 1.0$

Figure 3: A cross section of the MARS15 model of the tunnel with the first secondary collimator S1 (top) and a fragment of its core (bottom). 

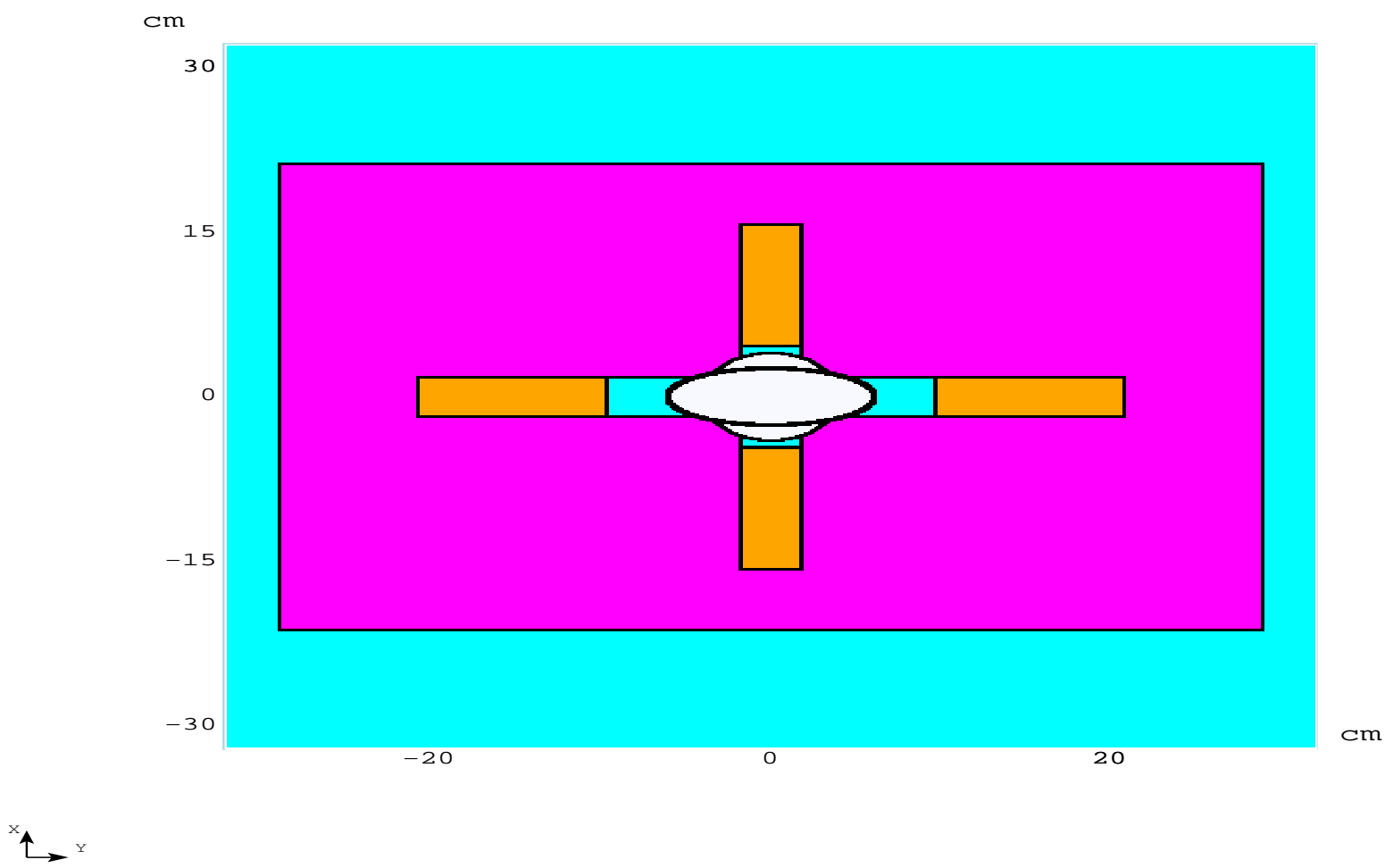

Aspect Ratio: $\mathrm{X}: \mathrm{Y}=1: 1.0$
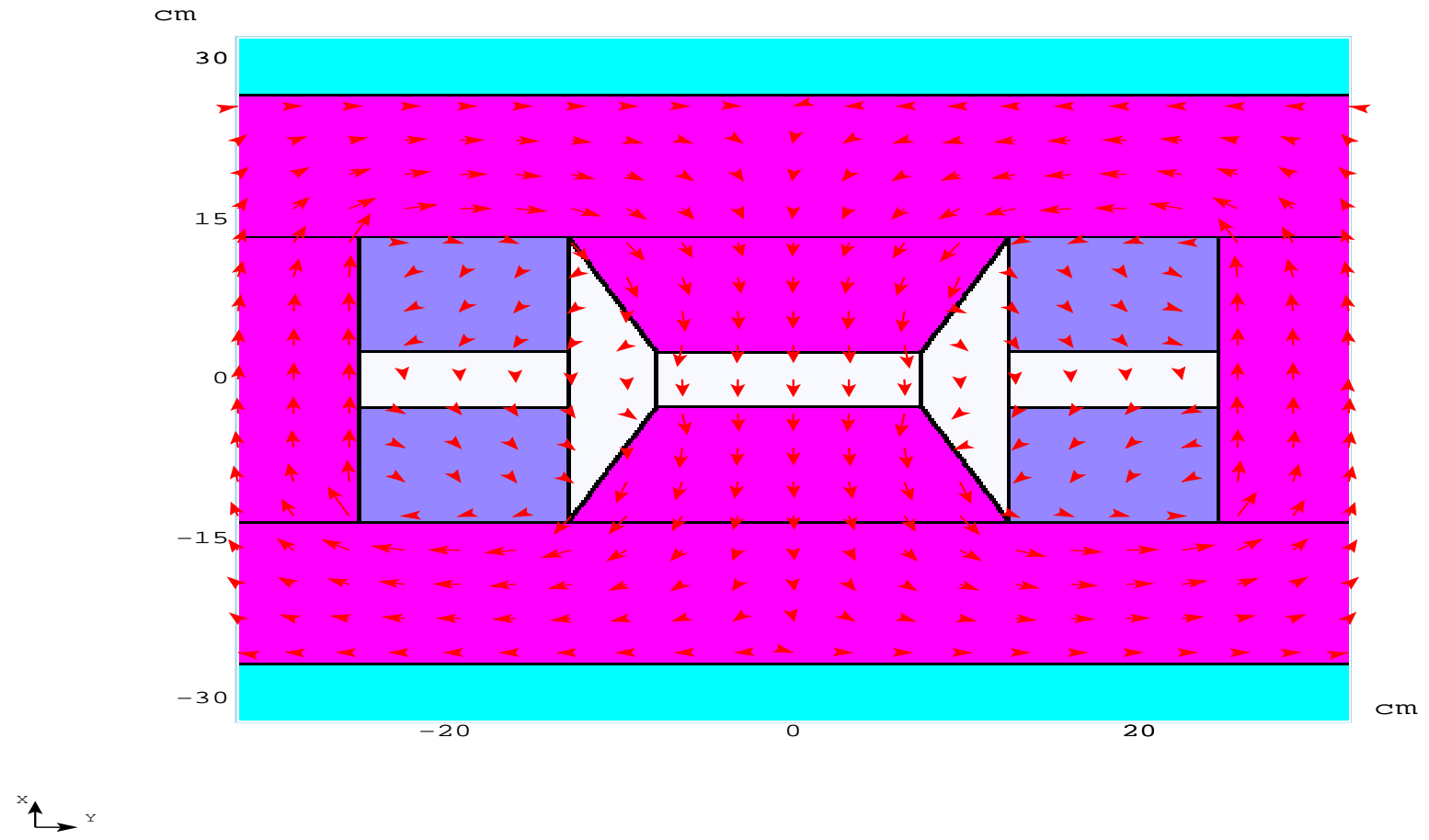

Figure 4: A cross section of the MARS15 model of a quadrupole (top) and dipole (bottom). Magnetic field is shown with arrows only for the dipole. 

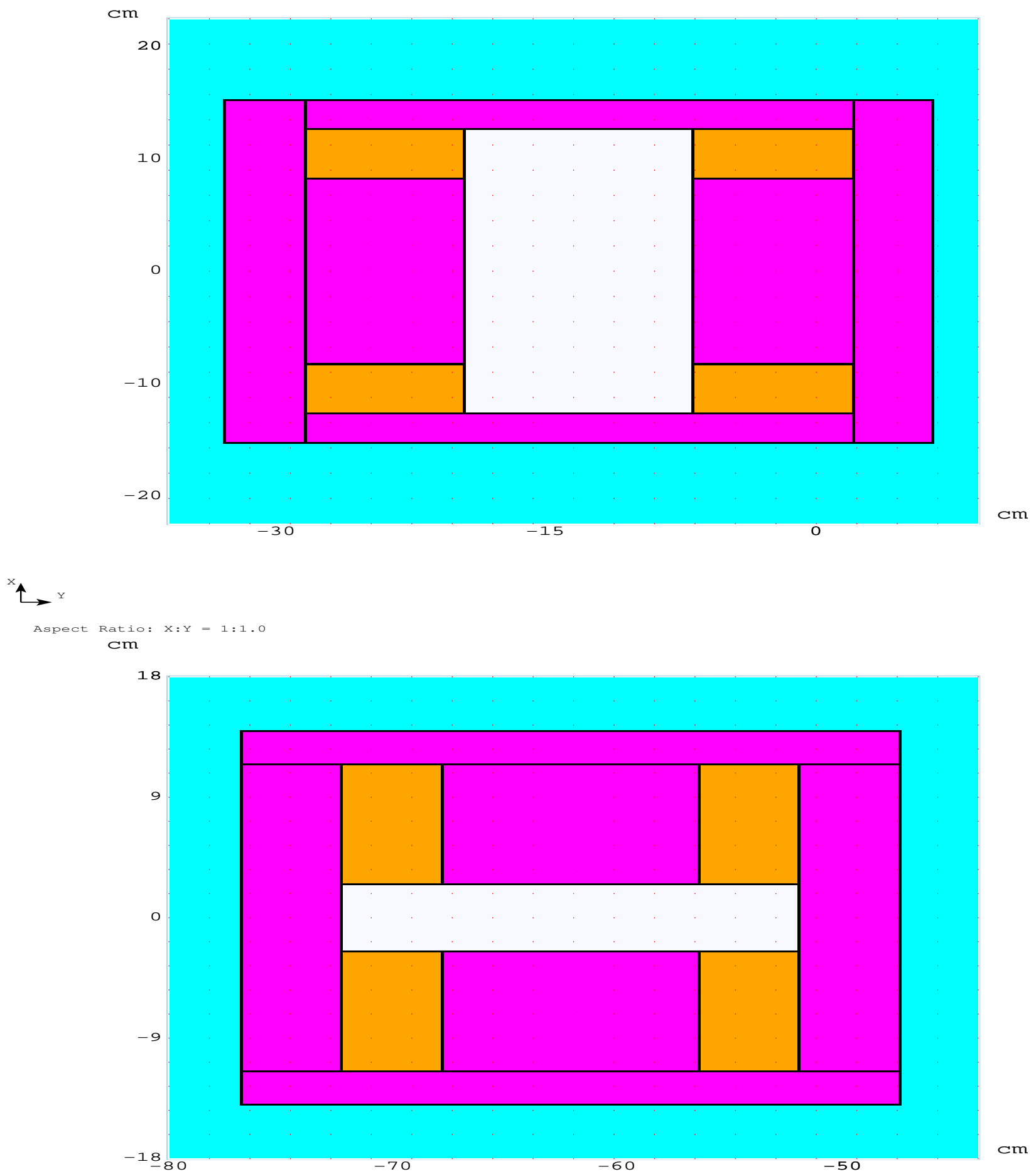

Figure 5: A cross section of the MARS15 model of a vertical trim dipole (top) and horizontal one (bottom). 
includes all essential elements with detailed description of geometry and materials [5]. Table 1 describes the longitudinal sequence of the elements in the beam line. Due to curvature of the beam line the path length, $\mathrm{S}$, differs from $Z$-coordinate shown in the Figures below (except for the case of Q230). The MI tunnel with concrete walls is surrounded by gravel, while there is clay under the floor. Detailed two-dimensional distributions of magnetic field in the dipoles and quadrupoles were calculated with the OPERA-2D code [6]. In order to identify materials used in the model, the following color scheme is employed:

- blue - air

- brown - magnet coil

- dark blue - marble

- green - soil

- grey - concrete

- light blue - polyethylene

- red - yoke (steel)

- turquoise - steel

- violet - copper

- white - vacuum

Each of the secondary collimators (see Figs. 2 and 3) has a steel core covered with marble layers to shield residual activity [7]. Only surfaces that are easy to reach are covered with marble: front, rear, left side, and left top. For each of the secondary collimators, the jaws were aligned precisely according to the results of the collimation studies [2, 7]. In addition, each of the collimators but S4 has two masks: a polyethylene mask upstream and steel/concrete mask downstream. The last collimator, S4, needs only the mask downstream. The masks are used to absorb radiation scattered in backward and forward direction and, therefore, provide extra protection.

An electron cooling system is installed in the region from Q305 up to Q307. In order to protect the sensitive equipment against scattered radiation, a concrete wall $(100 \mathrm{~cm}$ in length, $136 \mathrm{~cm}$ in width, and $152 \mathrm{~cm}$ in height) is required upstream of Q305 (see Fig. 2).

All quadrupoles in the region have the same design, so that the same geometry model is used (see Fig. 4). Only lengths and magnetic fields can differ. The same statements apply to bending dipoles in the region. There are several horizontal and vertical trim dipoles in the beam line (see Fig. 5). It is assumed that, during the slip-stacking injection, magnetic field in most trim dipoles is equal to zero [2]. Therefore, in the model the trim dipoles, each $30.5 \mathrm{~cm}$ in length, serve only for the purpose of absorption of scattered radiation.

In addition, the beam line includes also four horizontal trim dipoles with non-zero magnetic field upstream of the following quadrupoles: Q302, Q304, Q306, and Q308. 
Table 1: Beam line elements in the MI collimation region. The path length, $\mathrm{S}$, is calculated along the beam line and given for downstream end of each element. Primary collimator, masks, and the shielding wall in front of Q305 are not presented on the list.

\begin{tabular}{|c|c|c|c|c|c|}
\hline Element & Type of element & $\mathrm{S}(\mathrm{m})$ & Element & Type of element & $\mathrm{S}(\mathrm{m})$ \\
\hline Drift & & -2.95 & K304 & kicker & 87.14 \\
\hline Q230 & quadrupole & 0.00 & Drift & & 87.75 \\
\hline Drift & & 0.77 & H304 & trim dipole & 88.05 \\
\hline IDC011 & dipole & 4.83 & Drift & & 88.22 \\
\hline Drift & & 5.19 & Q304 & quadrupole & 90.35 \\
\hline IDD002 & dipole & 9.25 & Drift & & 105.04 \\
\hline Drift & & 9.58 & V305 & trim dipole & 105.34 \\
\hline V231 & trim dipole & 9.89 & Drift & & 105.51 \\
\hline Drift & & 10.02 & Q305 & quadrupole & 107.64 \\
\hline Q231 & quadrupole & 12.97 & Drift & & 122.33 \\
\hline Drift & & 13.73 & H306 & trim dipole & 122.63 \\
\hline IDC012 & dipole & 17.80 & Drift & & 122.79 \\
\hline Drift & & 18.15 & Q306 & quadrupole & 124.93 \\
\hline IDD010 & dipole & 22.22 & Drift & & 139.61 \\
\hline Drift & & 22.55 & V307 & trim dipole & 139.92 \\
\hline $\mathrm{H} 232$ & trim dipole & 22.85 & Drift & & 140.08 \\
\hline Drift & & 22.98 & Q307 & quadrupole & 142.22 \\
\hline Q232 & quadrupole & 25.93 & Drift & & 143.18 \\
\hline Drift & & 26.70 & S3 & collimator & 144.82 \\
\hline IDC019 & dipole & 30.76 & Drift & & 156.90 \\
\hline Drift & & 31.12 & H308 & trim dipole & 157.21 \\
\hline IDD024 & dipole & 35.18 & Drift & & 157.37 \\
\hline Drift & & 35.51 & Q308 & quadrupole & 159.50 \\
\hline V301 & trim dipole & 35.82 & Drift & & 167.47 \\
\hline Drift & & 35.95 & S4 & collimator & 169.11 \\
\hline Q301 & quadrupole & 38.49 & Drift & & 174.22 \\
\hline Drift & & 39.95 & V309 & trim dipole & 174.53 \\
\hline S1 & collimator & 41.59 & Drift & & 174.66 \\
\hline Drift & & 53.17 & Q309 & quadrupole & 177.20 \\
\hline H302 & trim dipole & 53.48 & Drift & & 177.96 \\
\hline Drift & & 53.64 & IDC042 & dipole & 182.03 \\
\hline Q302 & quadrupole & 55.78 & Drift & & 182.39 \\
\hline Drift & & 70.46 & IDD037 & dipole & 186.45 \\
\hline V303 & trim dipole & 70.77 & Drift & & 186.78 \\
\hline Drift & & 70.93 & H310 & trim dipole & 187.08 \\
\hline Q303 & quadrupole & 73.07 & Drift & & 187.22 \\
\hline Drift & & 74.03 & Q310 & quadrupole & 190.16 \\
\hline $\mathrm{S} 2$ & collimator & 75.67 & Drift & & 190.93 \\
\hline Drift & & 85.15 & & & \\
\hline
\end{tabular}


However, the beam line is built by means of the MMBLB with the magnetic field in these trim dipoles turned off. For subsequent calculations of the beam loss and radiation transport in the region, the magnetic field is turned on.

Some details of the geometry model, such as shape of the 1-m shielding block and angle between the block and tunnel wall (Fig. 2), may look odd. Such an erroneous perception is due to the following: (i) finite resolution of the figures; (ii) distorted aspect ratio because of different scaling factors applied in horizontal and vertical directions (even if the aspect ratio is shown as 1:1).

\section{Model of Beam Loss on the Collimators}

The two-stage collimation system designed for the slip-stacking injection in MI [2] comprises a single-jaw horizontal primary collimator (tungsten bar $0.25 \mathrm{~mm}$ in thickness placed between Q230 and IDC011 at Z=36 cm) and four secondary collimators S1 thru S4 with $5.08 \mathrm{~cm} \mathrm{X} 10.16 \mathrm{~cm}$ rectangular stainless steel apertures (Figs. 1, 2, and 3). A detailed description of alignment of the collimators is given in [2]. For the purpose of radiation shielding studies the beam loss on the collimators should be modeled as precisely as possible. Using the MARS code, there are two options to achieve the goal: (i) direct modeling starting from the primary collimator; (ii) using the spatial beam loss distribution on the secondary collimators calculated with the STRUCT code [2] as a source for subsequent Monte Carlo modeling of radiation transport with the MARS code. The first option is very sensitive to misalignment of the beam line elements in the model, details of the magnetic field distributions and so forth. Therefore, the second option is used in the study to determine the radiation distribution. The calculated data on integral beam loss on the secondary collimators is given in Table 2 . It should be noted that, according to the STRUCT results [2], $99.7 \%$ of total beam loss occurs inside the collimation region. $87.3 \%$ out of the $99.7 \%$ occurs on the four secondary collimators while the remaining $12.4 \%$ is lost on the beam pipe and other elements. In order to perform conservative calculations, one assumes that $100 \%$ of the beam loss occurs on the four secondary collimators.

Table 2: Breakdown of the calculated integral beam loss on the secondary collimators. Here $100 \%$ corresponds to $1.25 \times 10^{12}$ protons lost per second.

\begin{tabular}{|c|c|}
\hline Collimator & Beam loss (\%) \\
\hline S1 & 28 \\
S2 & 40 \\
S3 & 10 \\
S4 & 22 \\
\hline Total & 100 \\
\hline
\end{tabular}




\section{Results of Calculations}

\subsection{Surface water activation}

Activation of water with radionuclides is strictly limited by environmental protection regulations. In the calculations of surface water activation one follows the procedure developed at Fermilab and referred to as Fermilab Concentration Model (see [8, 9] and references therein). The procedure consists in determining the average star density over a so-called 99\%-volume of soil surrounding the tunnel. The volume should be big enough in order to satisfy the following condition: the calculated star density on external boundary of the volume should be at the level of $1 \%$ when compared to the calculated maximum star density on internal boundary, i.e. tunnel-soil interface. Either maximum star density at the tunnel-soil interface, $S_{\max }$, or star density averaged over such a $99 \%$-volume, $\langle S\rangle$, can be used with the Concentration Model. Numerous studies showed that the following simple dependence holds: $\langle S\rangle=S_{\max } / 50$, where the factor of 50 was confirmed in various independent calculations*.

The calculated distributions of star density around the region are shown in Fig. 6. The model of beam loss described in the previous section eventually gives rise to the star density distribution from S1 up to Q310. In order to calculate the distribution upstream of the latter region, i.e. from Q230 up to Q301, one needs to consider primary collimator as the initial source of beam loss. A separate calculation was performed with such a source for the region from Q230 up to Q301 and the star density distribution is also shown in Fig. 6. The highest calculated star densities are observed around the secondary collimators S1, S2, and S4. The distributions around the hottest spot-collimator S2-are shown in Fig. 7. The corresponding 99\%-volumes around the collimators are shown in Fig. 1. Vertical and horizontal dimensions of the volumes are approximately the same. The calculated average star densities are given in Table 3. There are underdrains all along the MI tunnel and suitably placed sump pumps and generation of radionuclides in surface water is of primary concern. Taking into account a realistic operational schedule and operational efficiency, annual amount of protons is expected to be $3.7 \times 10^{20}$ [10]. In such a case after a year of operation the Concentration Model gives rise to the following activation around the hottest spot (secondary collimator S2): surface water - 32\% of total limit; ground water (aquifer) much less than $1 \%$ of total limit.

Table 3: The calculated star densities $\left\langle S_{n}>\left(\mathrm{cm}^{-3} \mathrm{~s}^{-1}\right)\right.$ averaged over corresponding 99\%volumes around the secondary collimators $\mathrm{S} 1, \mathrm{~S} 2$, and S4.

\begin{tabular}{|l|l|}
\hline$<S_{1}>$ & 330 \\
$<S_{2}>$ & 460 \\
$<S_{4}>$ & 280 \\
\hline
\end{tabular}

\footnotetext{
${ }^{*} S_{\max }$ and $<S>$ were calculated independently in this study.
} 

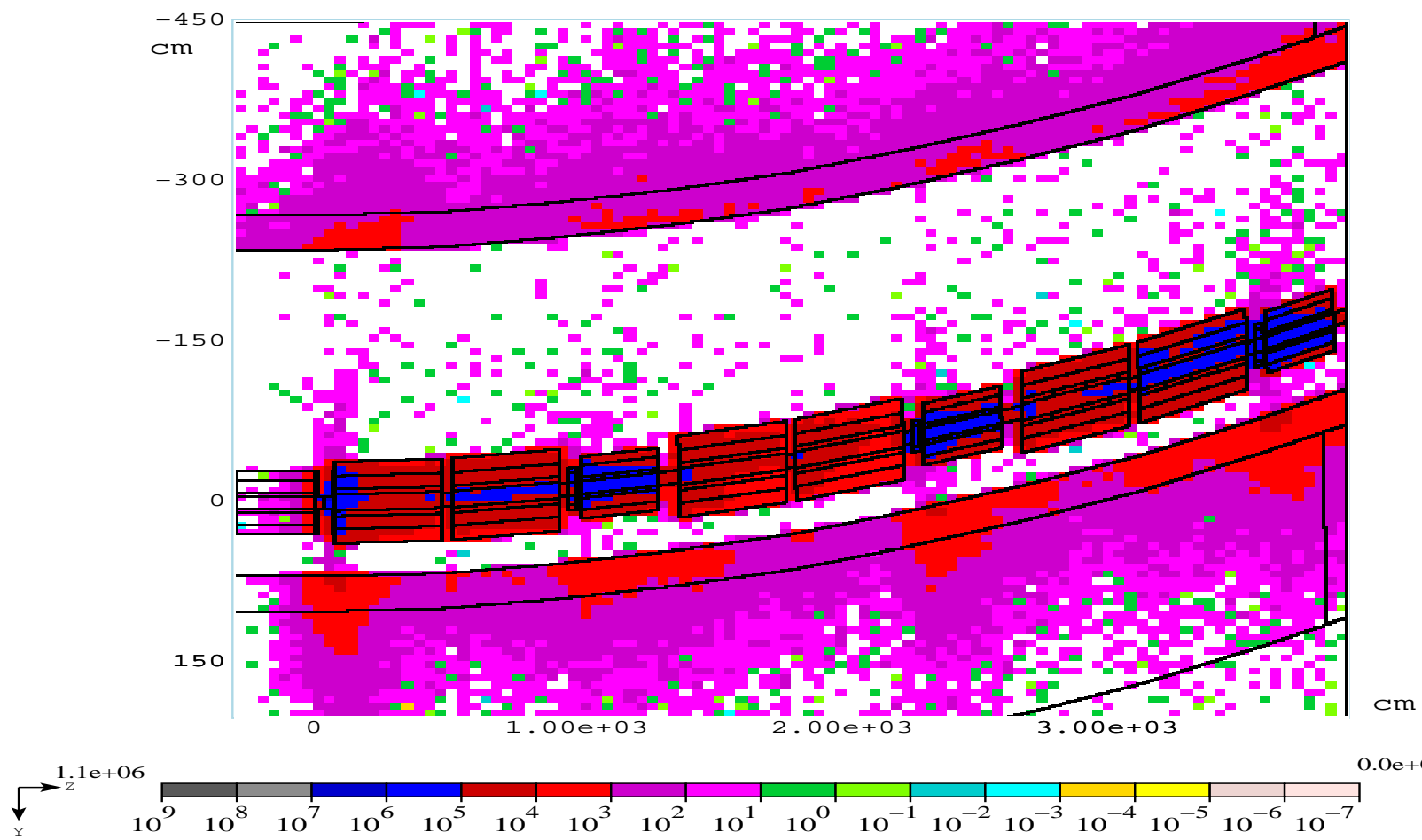
O.Oe+OO

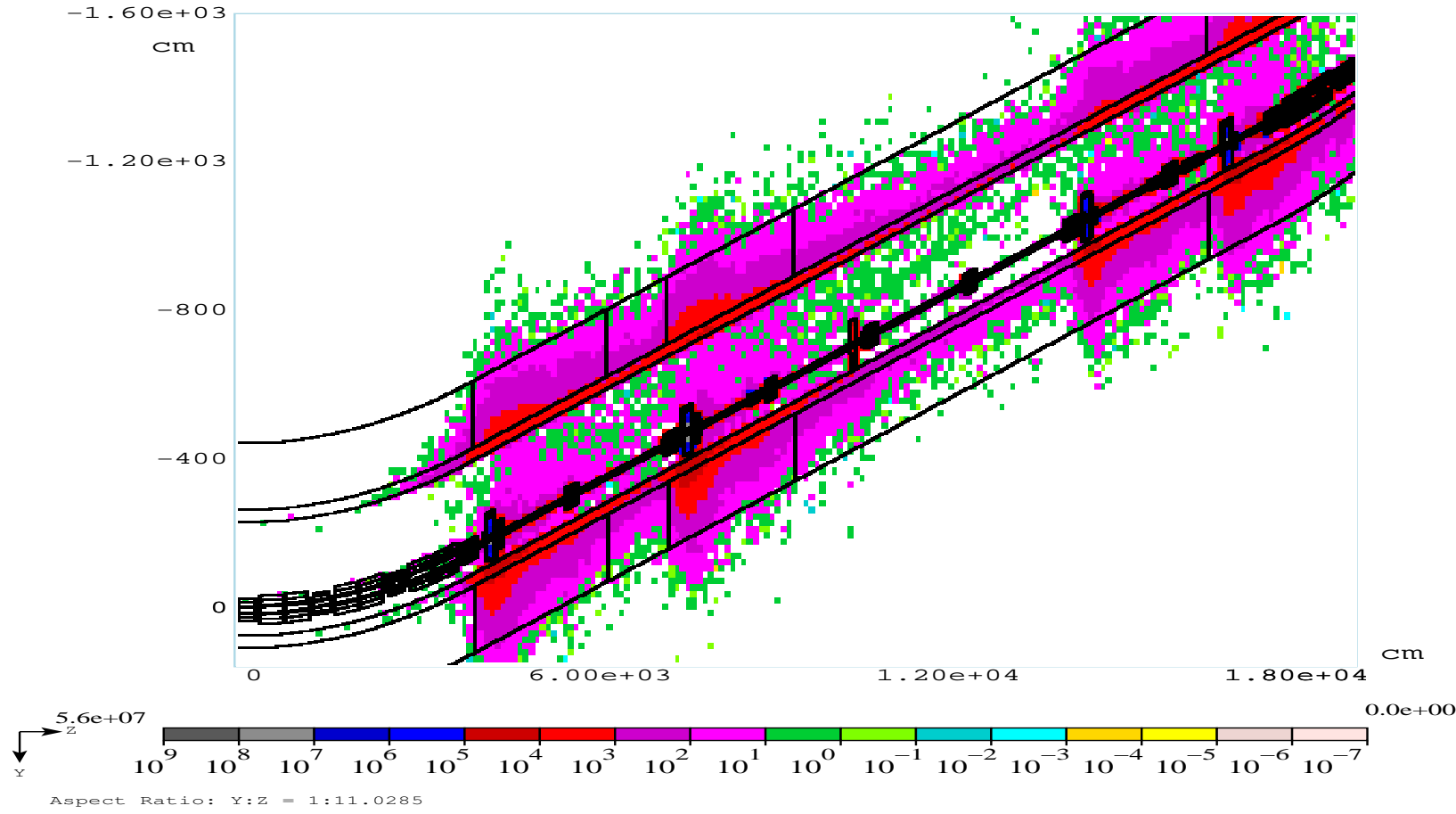

Figure 6: The calculated distributions (plan view) of star density $\left(\mathrm{cm}^{-3} \mathrm{~s}^{-1}\right)$ in the region from Q230 up to Q301 (top) and from S1 up to Q310 (bottom). 

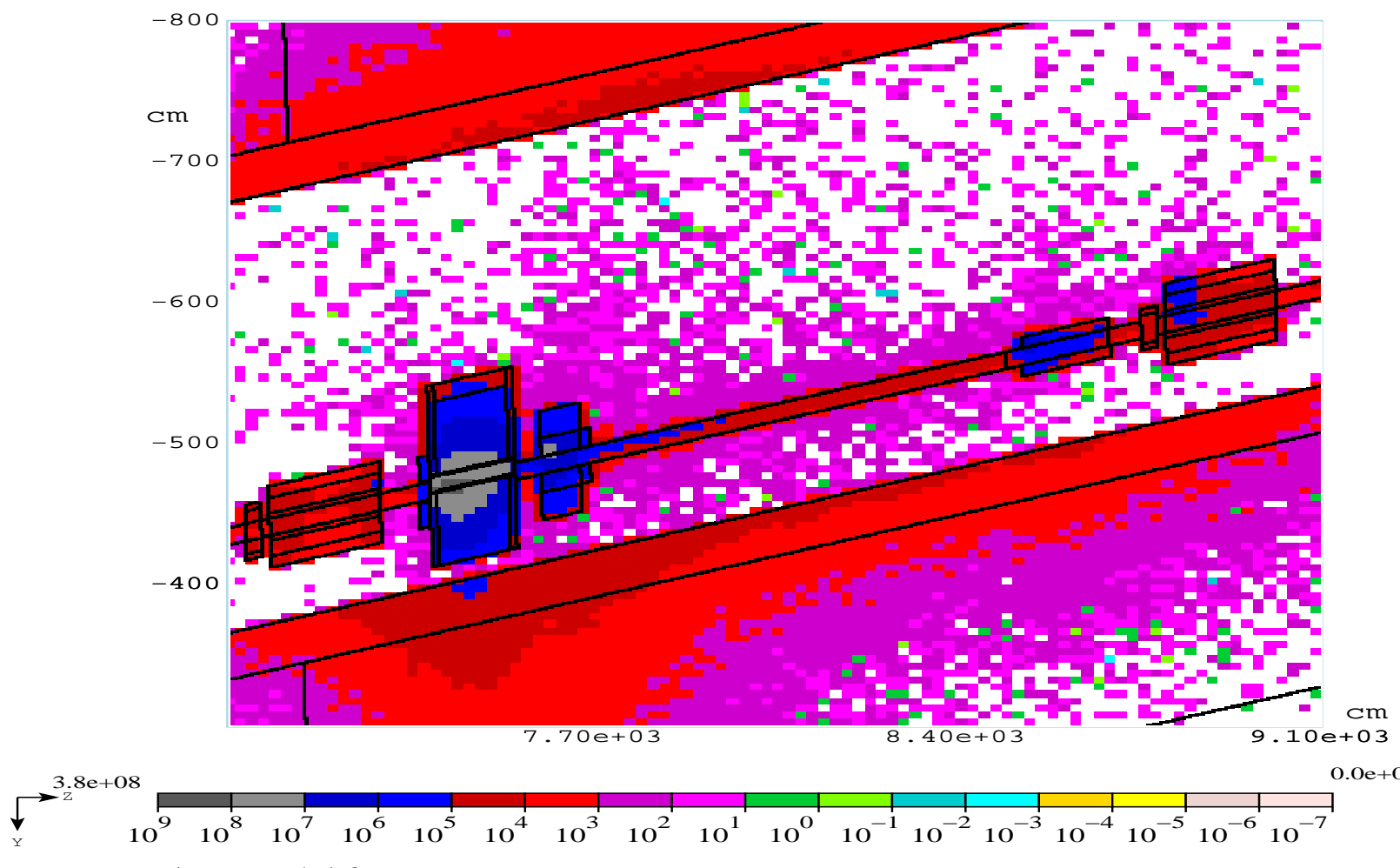

$\mathrm{O} . \mathrm{Oe}+\mathrm{OO}$
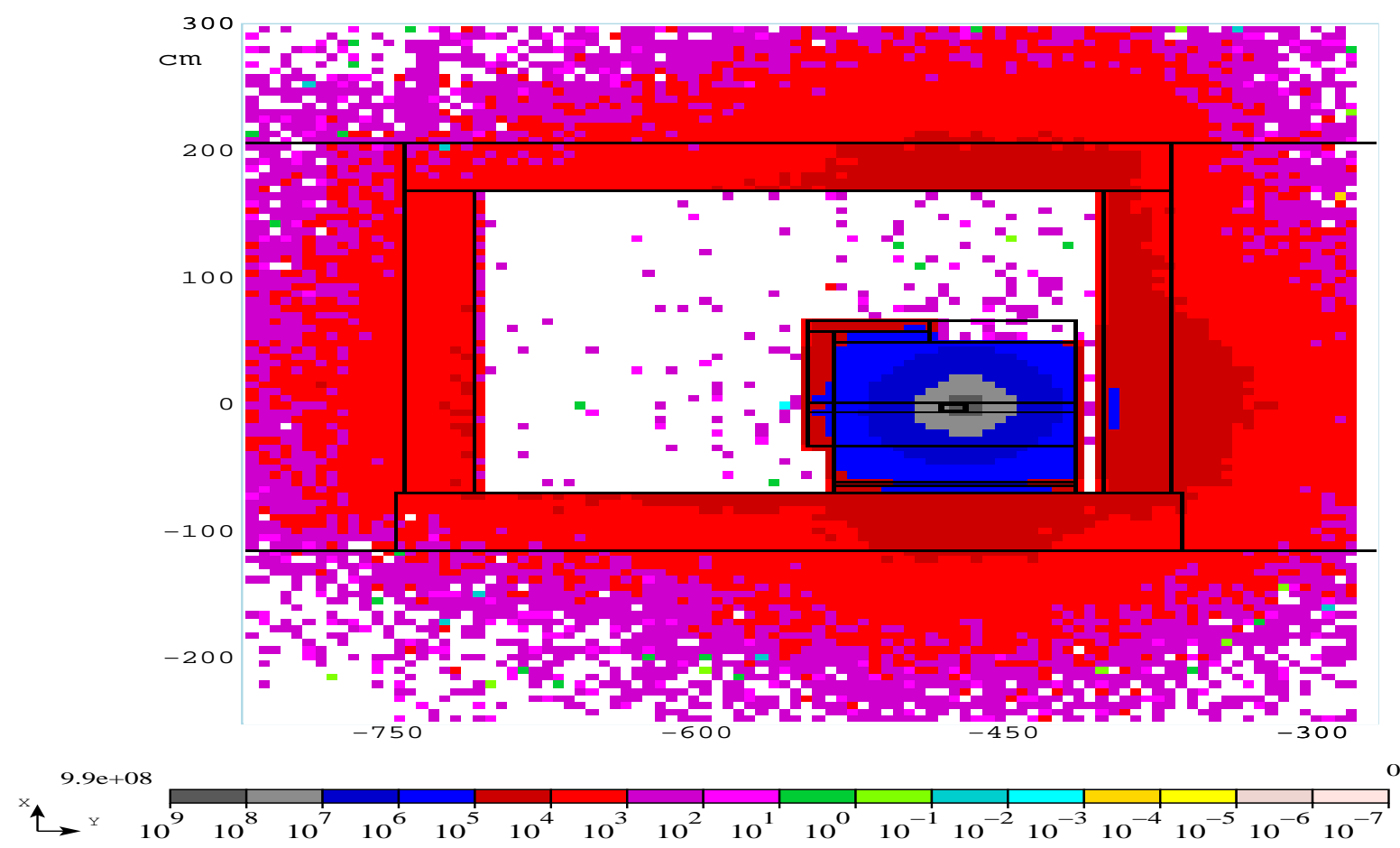

Aspect Ratio: $\mathrm{X}: \mathrm{Y}=1: 1.0$

Figure 7: The calculated distributions of star density $\left(\mathrm{cm}^{-3} \mathrm{~s}^{-1}\right)$ around the hottest spotcollimator S2: plan view (top) and cross section (bottom). 


\subsection{Dose load to magnet coils}

The calculated distributions of absorbed dose in the region are shown in Figs. 8 thru 10.
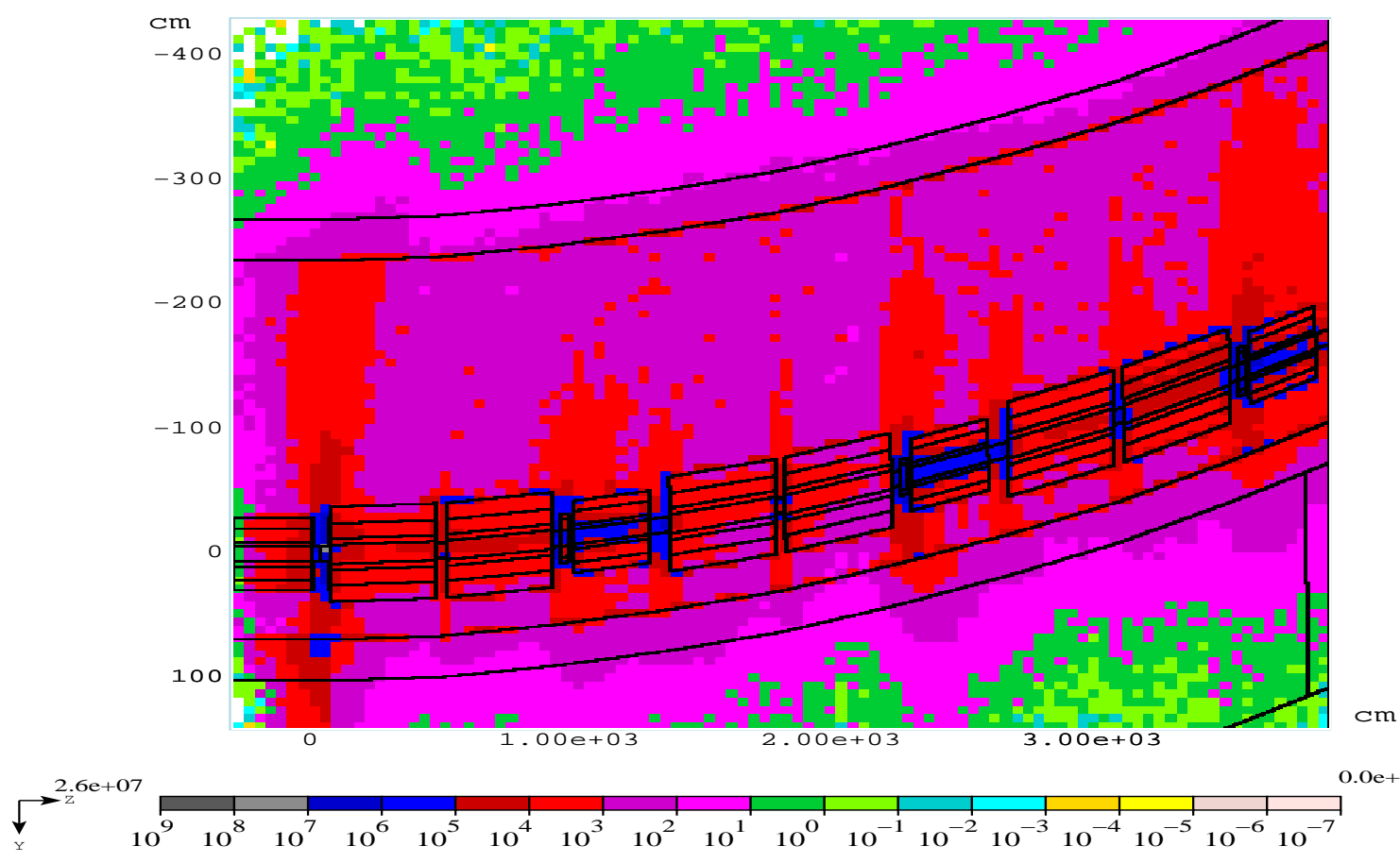

O.Oe+OO

Aspect Ratio: $Y: Z=1: 7.36842$
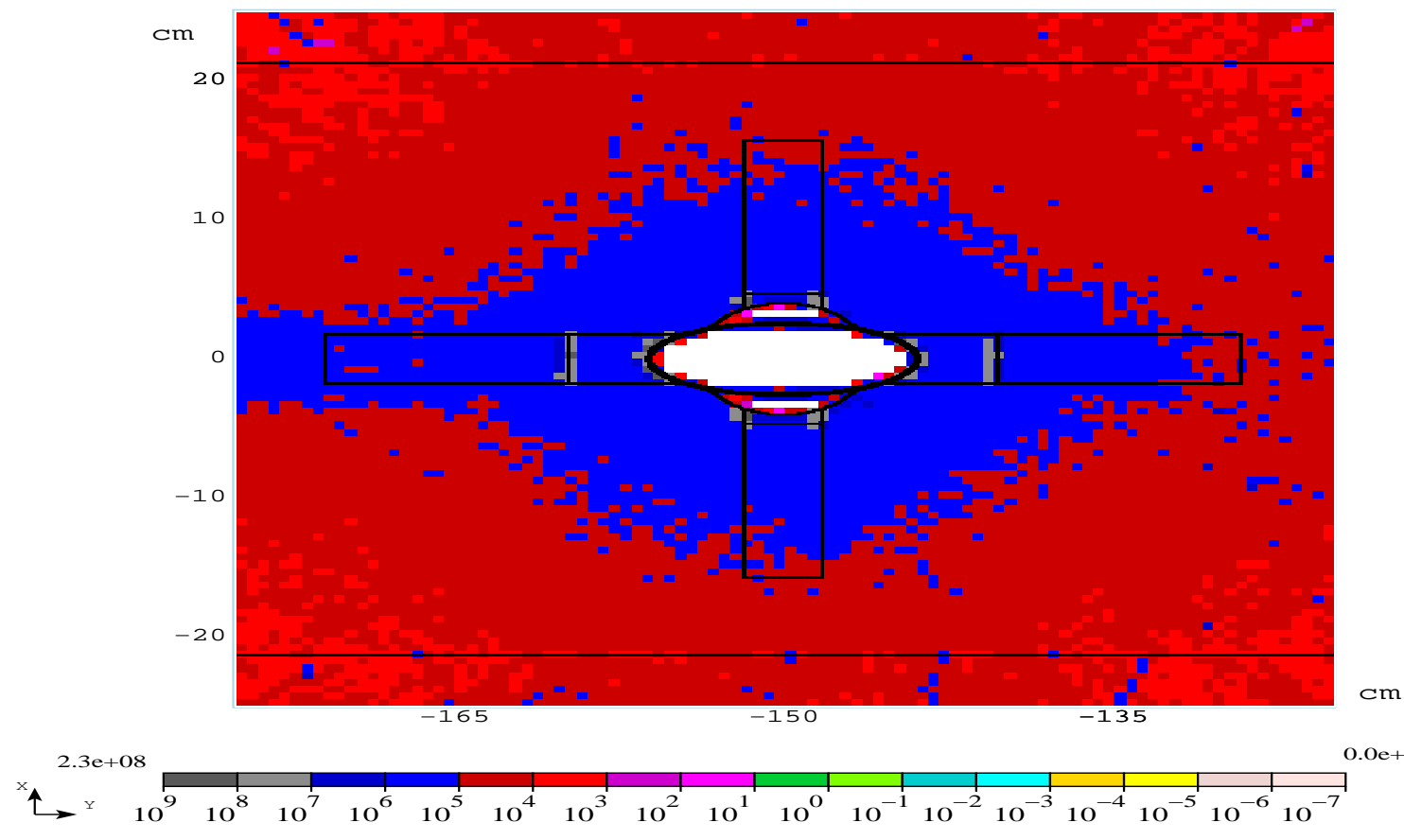

Aspect Ratio: $\mathrm{X}: \mathrm{Y}=1: 1$.0

Figure 8: The calculated distributions of absorbed dose (Gy/yr) from Q230 up to Q301 (plan view, top) and over the hottest spot-Q301 (cross section, bottom). 

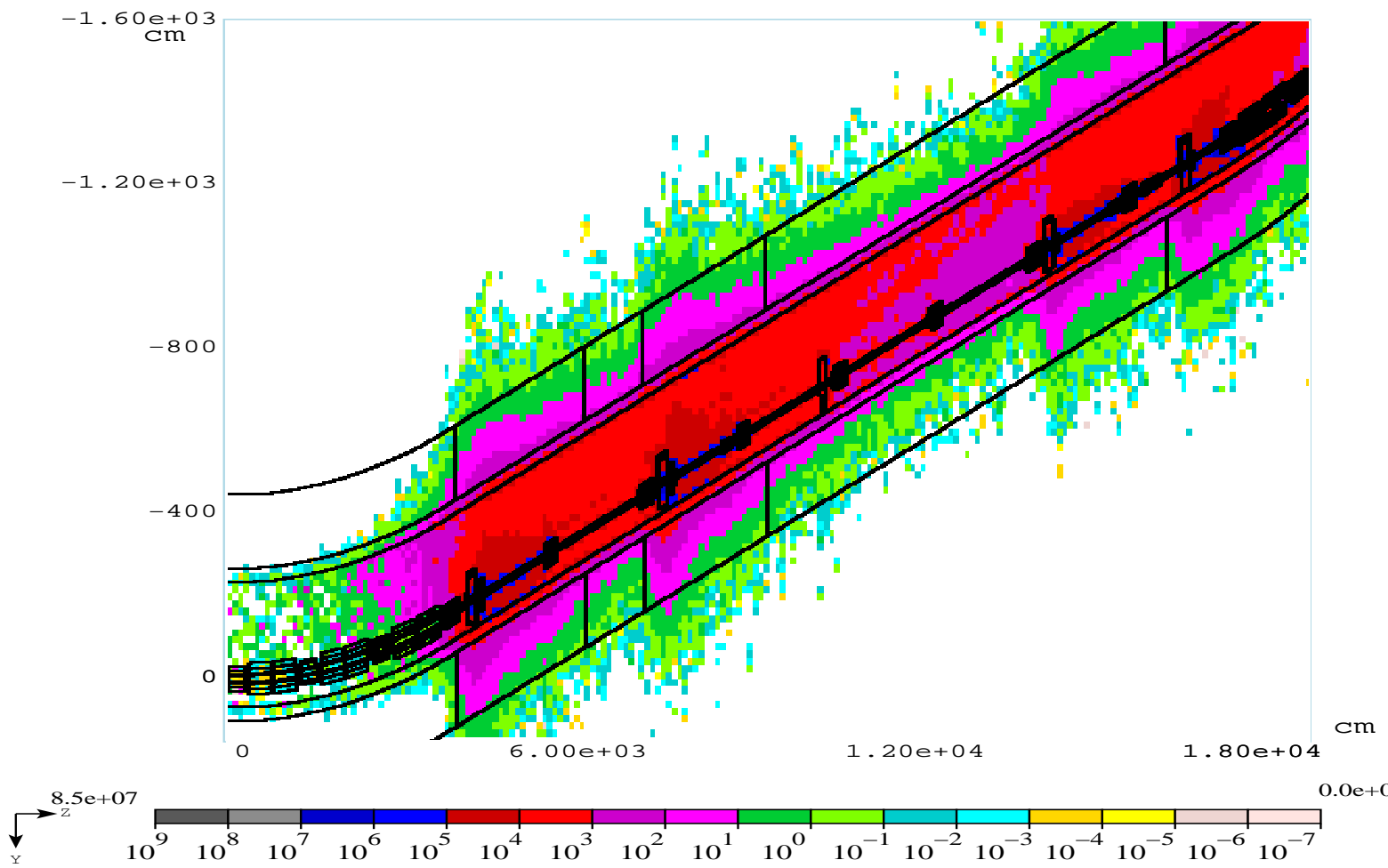

Aspect Ratio: $Y: Z=1: 11.0285$
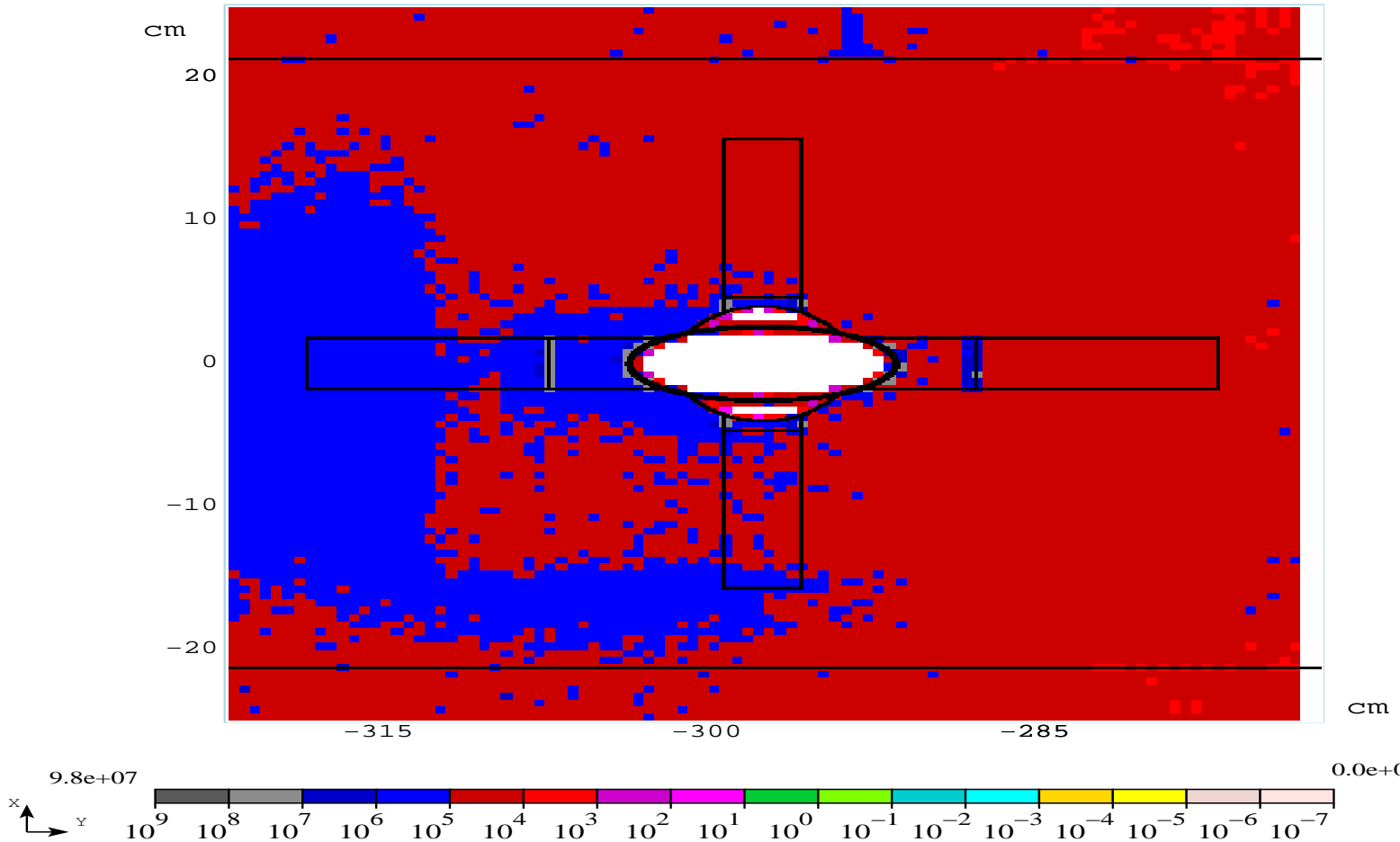

O.Oe+OO

Aspect Ratio: X:Y $=1: 1.0$

Figure 9: The calculated distributions of absorbed dose (Gy/yr) from the first secondary collimator S1 up to Q310 (plan view, top) and for Q302, the first quadrupole downsteram of $\mathrm{S} 1$ (cross section, bottom). 

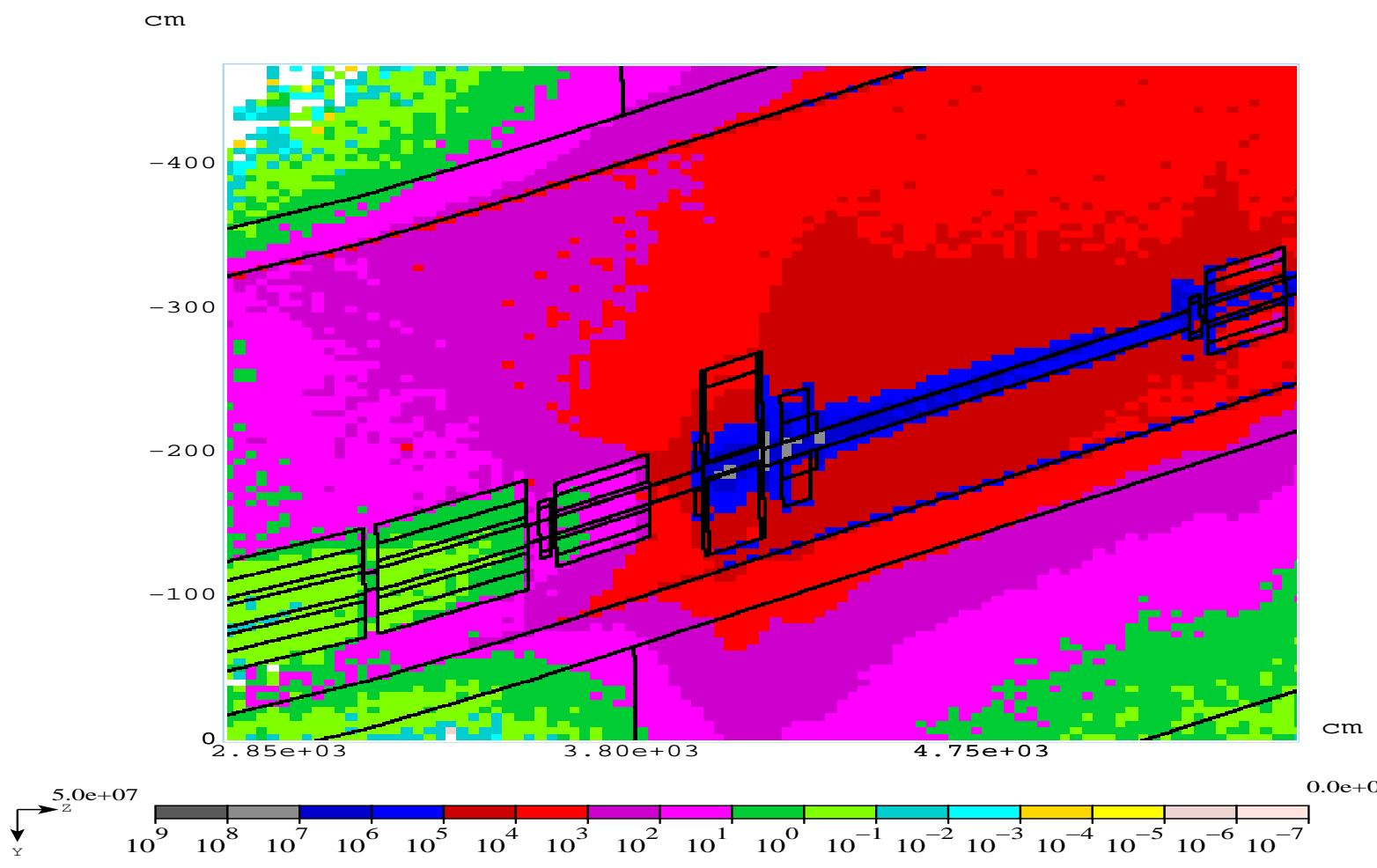

$0.0 e+00$
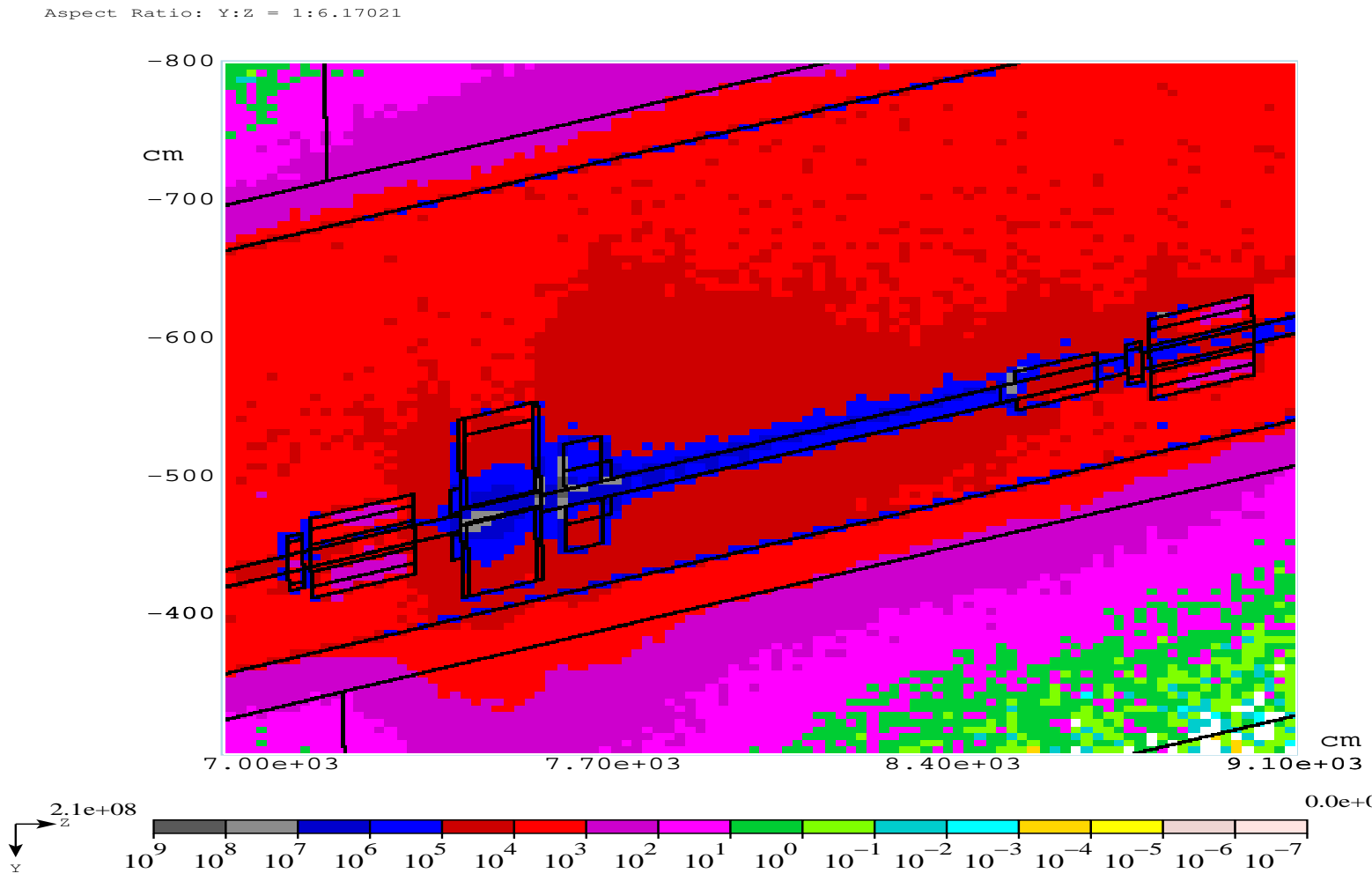

$\mathrm{O} . \mathrm{Oe}+\mathrm{OO}$

Aspect Ratio: $Y: Z=1: 4.4$

Figure 10: The calculated distributions (plan view) of absorbed dose (Gy/yr) around the first secondary collimator S1 (top) and around the second one S2 (bottom). 
Lifetime of a magnet depends to a great extent on the dose accumulated in its coils. The most vulnerable material in the coils is epoxy which can stand absorbed dose up to 4 MGy [11]. The highest dose — up to 1 MGy per year-is observed in coils of the Q301 quadrupole which is upstream of the secondary collimator S1. For the magnet this means the lifetime of about four years. For all the other dipoles, trim dipoles, and quadrupoles the absorbed dose in the hottest spot varies from 0.1 up to $0.5 \mathrm{MGy} / \mathrm{yr}$ which means the lifetime of about 8 years and more.

For the reasons described above (see previous section), the distributions for the regions from Q230 up to Q301 and from S1 up to Q310 were obtained from two separate calculations. Two marble masks, each $10 \mathrm{~cm}$ in thickness, are placed on downstream face of the quadrupole Q230 and upstream face of the dipole IDC011. The masks protect coils against radiation scattered on the primary collimator and backscattered from the dipole IDC011 as well as enable us to reduce residual activation of the magnets (see next section).

In Figs. 9 and 10 one can see the extended tails in forward direction due to scattering on the secondary collimators as well as significant backscattered component. In order to reduce the radiation scattered in forward direction (mostly low-energy neutrons shown in Fig. 11), masks consisting of a steel core surrounded with concrete (total thickness of $75 \mathrm{~cm}$ ) are placed downstream of the secondary collimators (Fig. 2). As to the absorbed dose, such a steel and concrete mask provides an extra absorption factor of about four. In response to these calculations (but not included in this report) an additional mask was placed downstream of S1, S2 and S4 but just upstream of the next machine element (H302, K304, V309) as described in Ref. [12]. Intensity of the backscattered radiation from the collimators can be reduced by means of the polyethylene masks $18 \mathrm{~cm}$ in thickness placed upstream of the collimators (Fig. 2). The polyethylene masks provide an extra absorption factor of about three (Fig. 11, right).
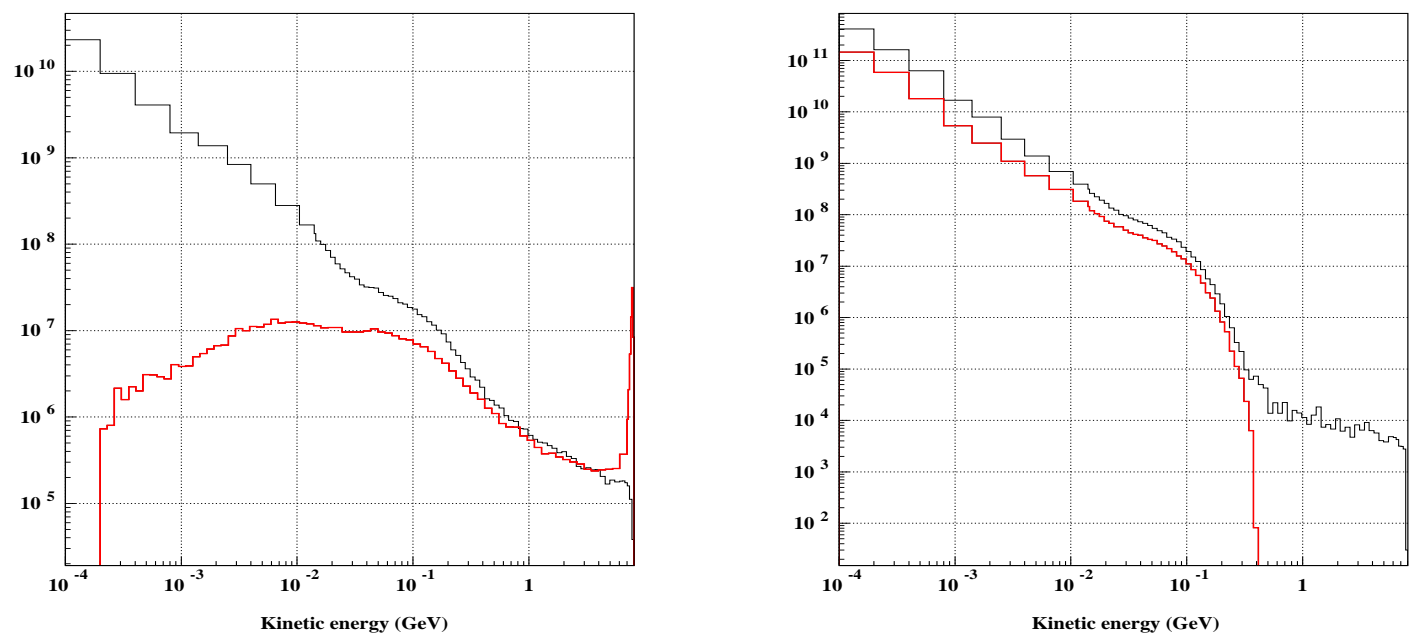

Figure 11: The calculated particle spectra $\left(\mathrm{GeV}^{-1} \mathrm{~cm}^{-2} \mathrm{~s}^{-1}\right)$ : (i) neutrons (black) and protons (red) between the secondary collimator S1 and its steel and concrete mask (left); (ii) neutrons between the quadrupole Q301 and collimator S1 (right) with (red) and without (black) the polyethylene mask on the upstream face of $\mathrm{S} 1$. 


\subsection{Distribution of residual activity}

Restrictions on residual activation of beam line components and tunnel are derived mostly from practical considerations. When planning on various maintenance and hands-on procedures, usually the residual dose is not to exceed $100 \mathrm{mrem} / \mathrm{hr}$ at a distance of $30 \mathrm{~cm}$ from the surface after a 30-day irradiation and 1-day cooling [11]. The calculated distributions of residual dose for the regions from Q230 up to Q301 and from S1 up to Q310 are shown in Figs. 12 thru 15 for a 30-day irradiation followed by a 1-day cooling.

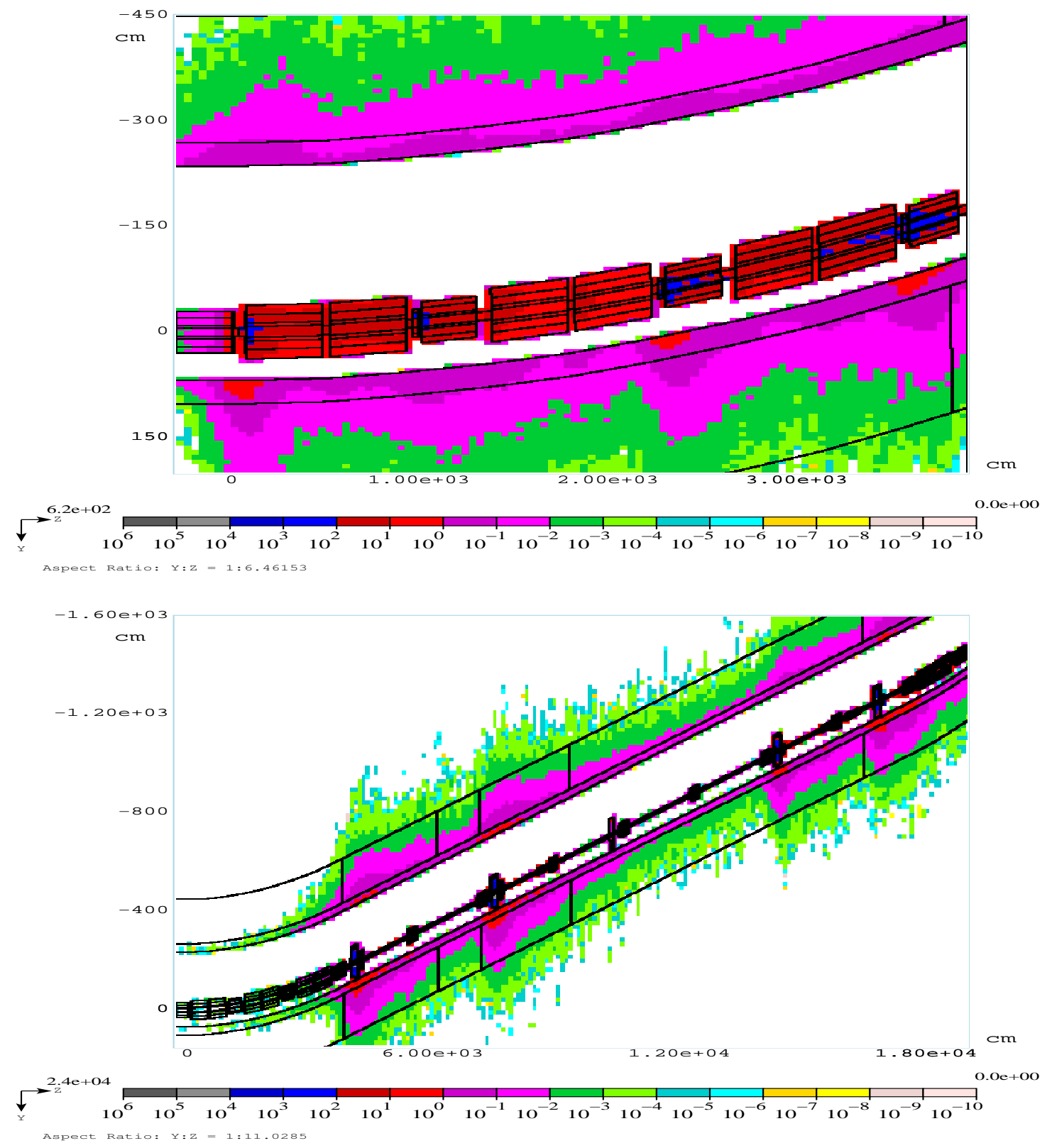

Figure 12: The calculated distributions (plan view) of contact residual dose ( $\mathrm{mrem} / \mathrm{hr}$ ) in the region from Q230 up to Q301 (top) and from S1 up to Q310 (bottom). 


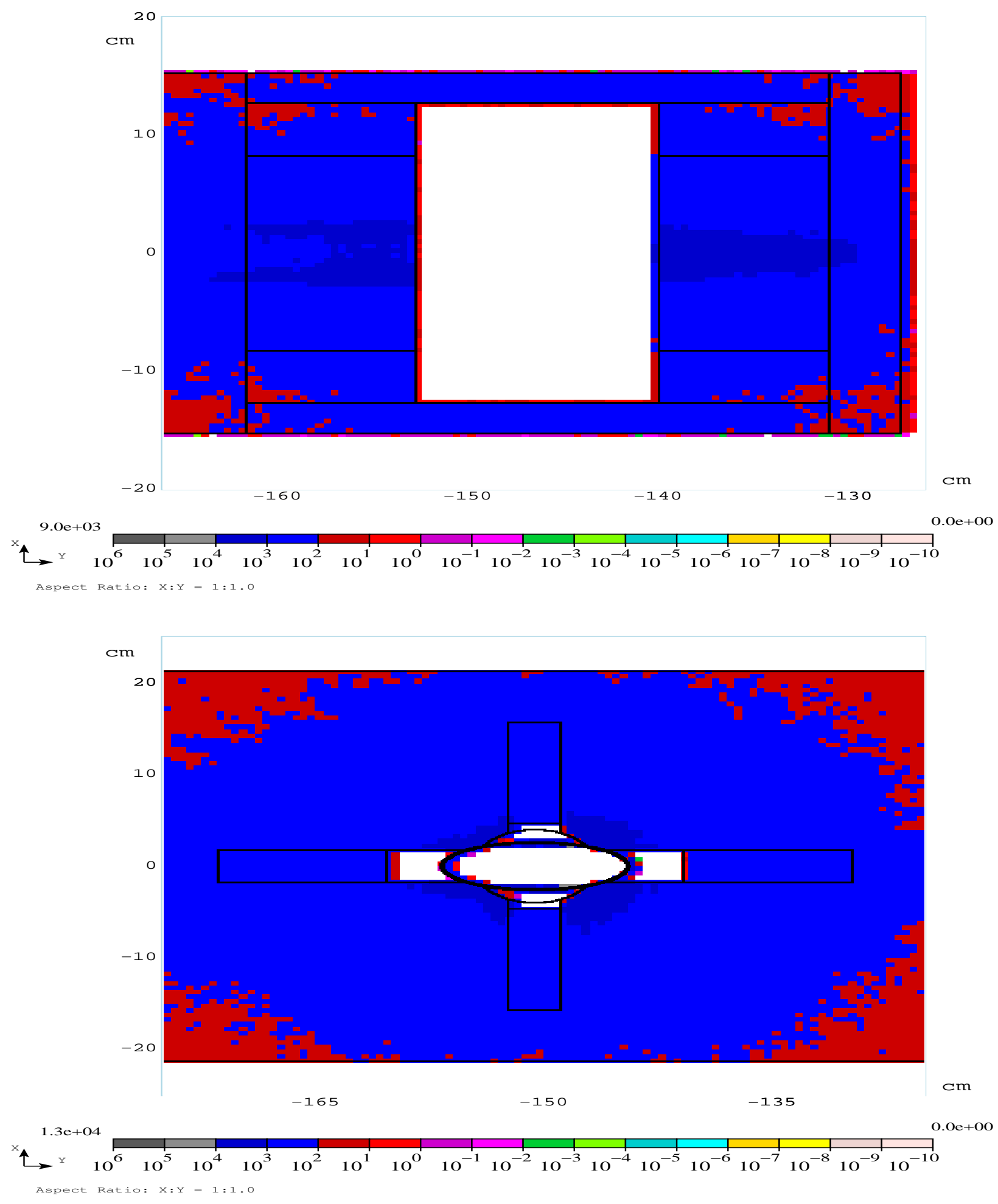

Figure 13: The calculated distributions (cross section) of contact residual dose (mrem/hr) for the trim dipole V301 (top) and quadrupole Q301 (bottom). 

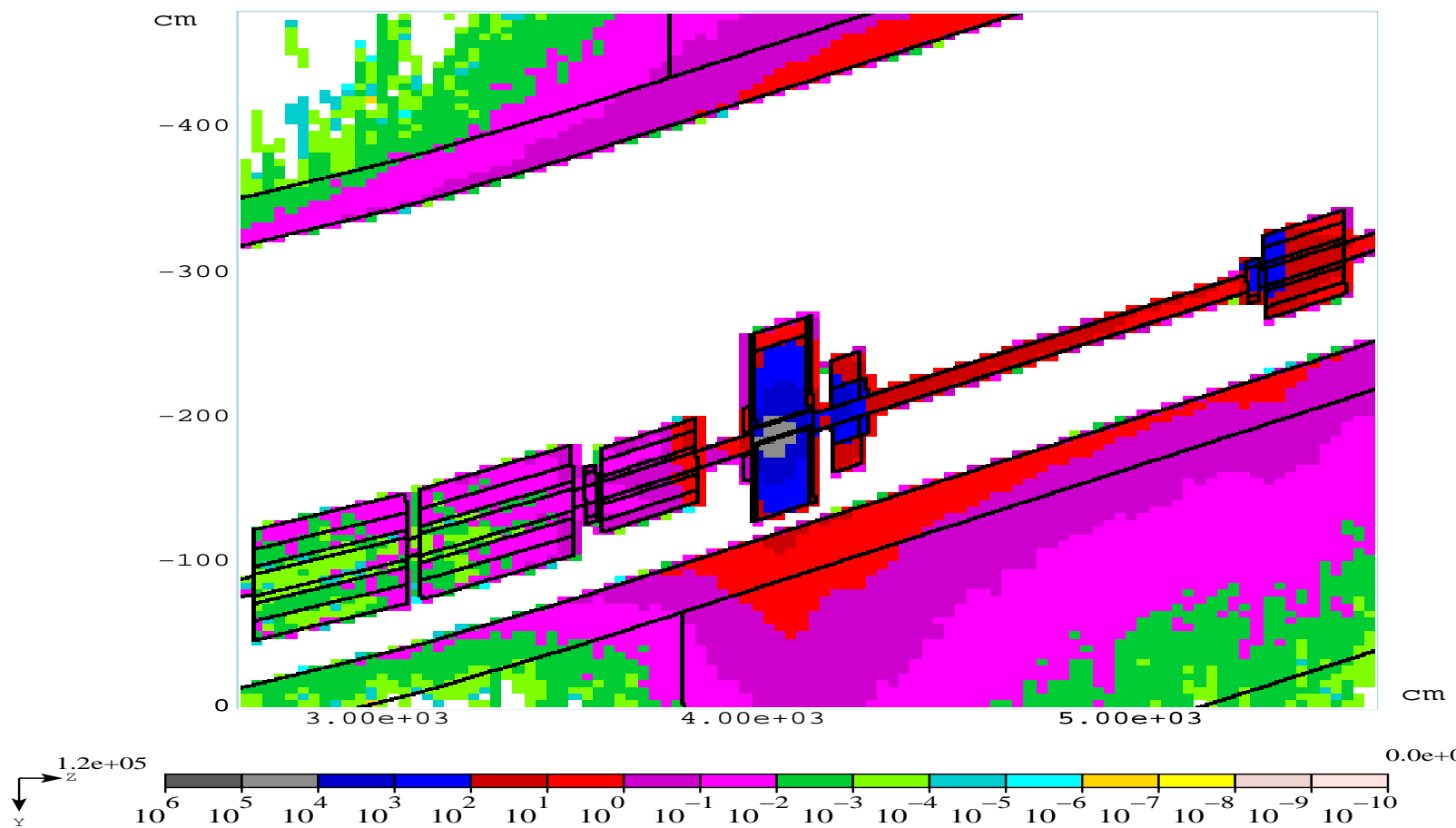

O.Oe+OO

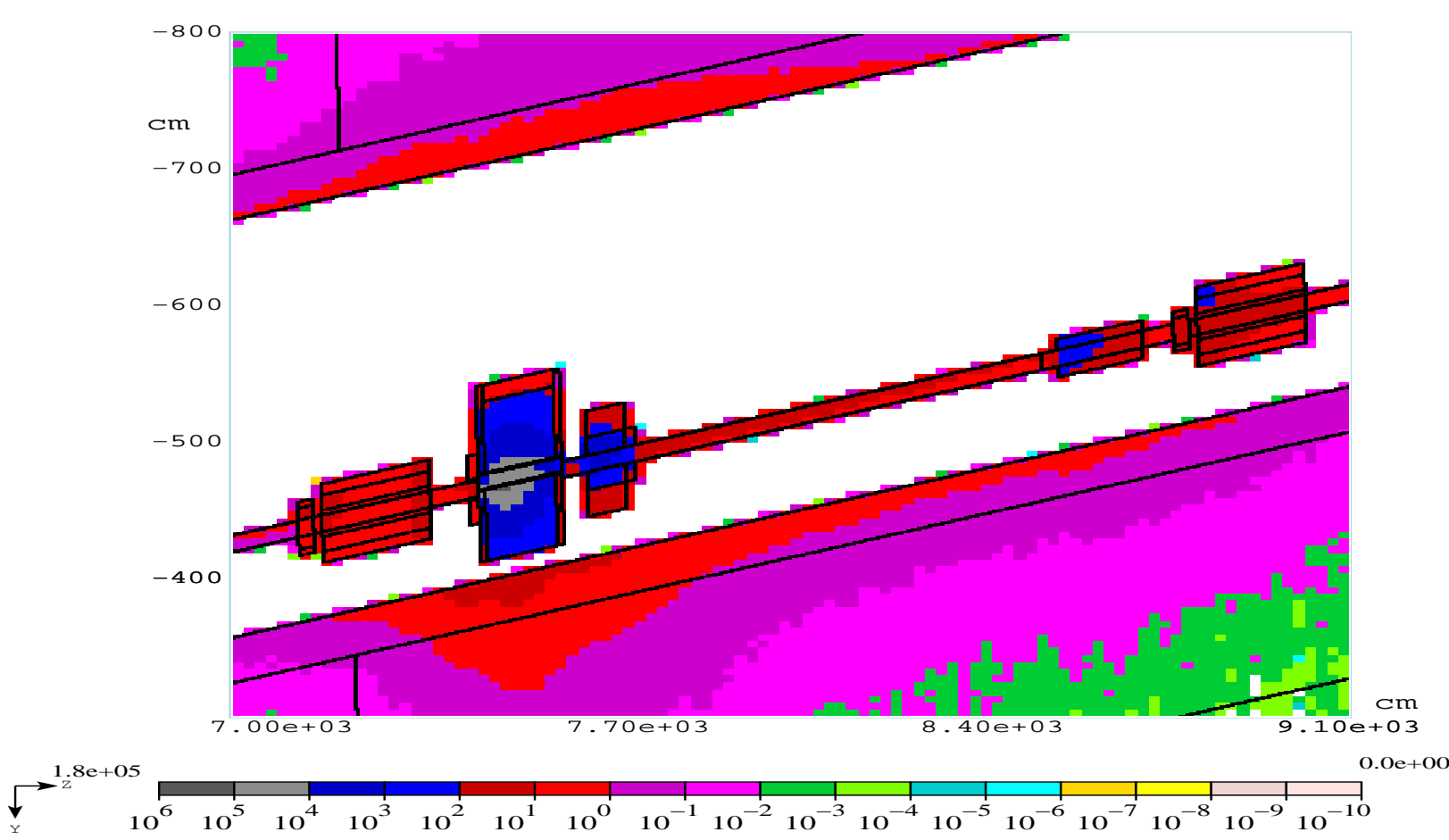

Figure 14: The calculated distributions (plan view) of contact residual dose $(\mathrm{mrem} / \mathrm{hr})$ around the first, S1 (top) and second, S2 (bottom) secondary collimator. 


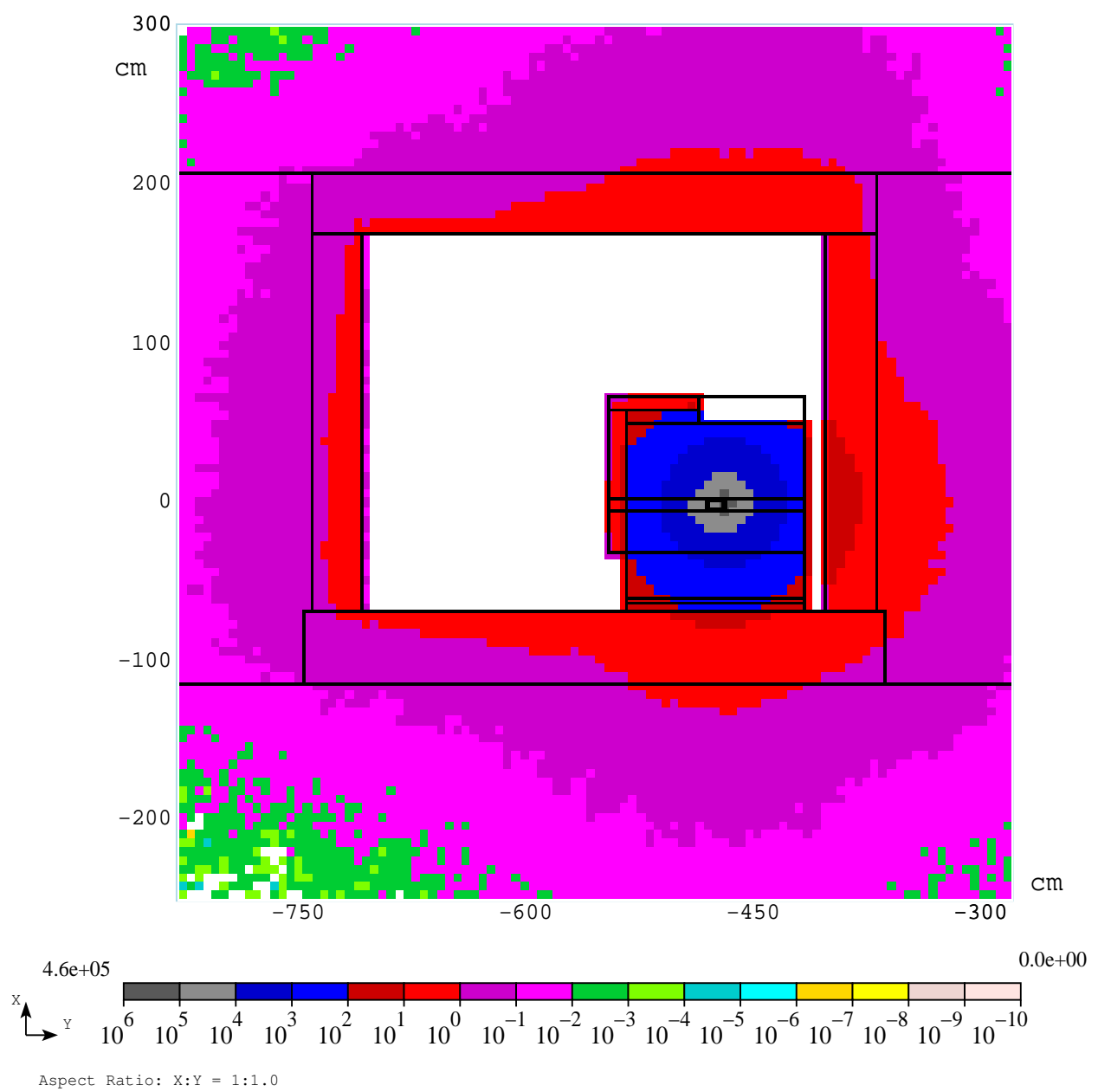

Figure 15: The calculated distribution (cross section) of contact residual dose ( $\mathrm{mrem} / \mathrm{hr}$ ) around the shower maximum in the secondary collimator S2.

All the distributions in this section are given for standard conditions: a 30-day irradiation followed by 1-day cooling. There are several small-with linear dimension of about $10 \mathrm{~cm}$ - hot spots around Q232, V301, Q301, and Q302. The spots reveal residual dose above $1 \mathrm{rem} / \mathrm{hr}$ and the highest dose is observed around the beam pipe (Fig. 13). Provided the distance between the magnets in the region from Q230 up to Q301 is small, the issue of high residual activity can be resolved by means of a local shielding such as bags with poly-beads, sand etc.

About $70 \%$ of the total beam loss in the collimation region occurs on the first two secondary collimators, S1 and S2. As a consequence, the hottest spots are observed inside the collimators (Fig. 14). In order to reduce the contact residual dose on the surface of the collimators down to an acceptable level, the bodies of all the secondary collimators-front, rear, and part of side surface-are covered with marble layers $10 \mathrm{~cm}$ in thickness (Figs. 2 and 3). The advantage of using marble is in its extremely low residual activation while providing significant absorption of $\mathrm{MeV}$ gammas generated in the steel core [7]. One can 
see from Fig. 15 that the hottest spots around S2 are the following: (i) $70 \mathrm{mrem} / \mathrm{hr}$ on the wall at the beam line elevation $(\mathrm{X}=0)$; (ii) $110 \mathrm{mrem} / \mathrm{hr}$ atop the $\mathrm{S} 2$ itself.

For the hottest spots on the aisle side of the beam line-V301, Q301, H304 and S2cooling curves are provided in Fig. 16. For the hottest spots on the aisle side of the collimators S1 and S2 the contact residual dose after a 1-year irradiation and 1-hr cooling is approximately 8 times the $30 \mathrm{~d}+1 \mathrm{~d}$ residual dose. For the hottest spots on the left wall near the collimators (see Fig. 15) the corresponding factor is about 4.

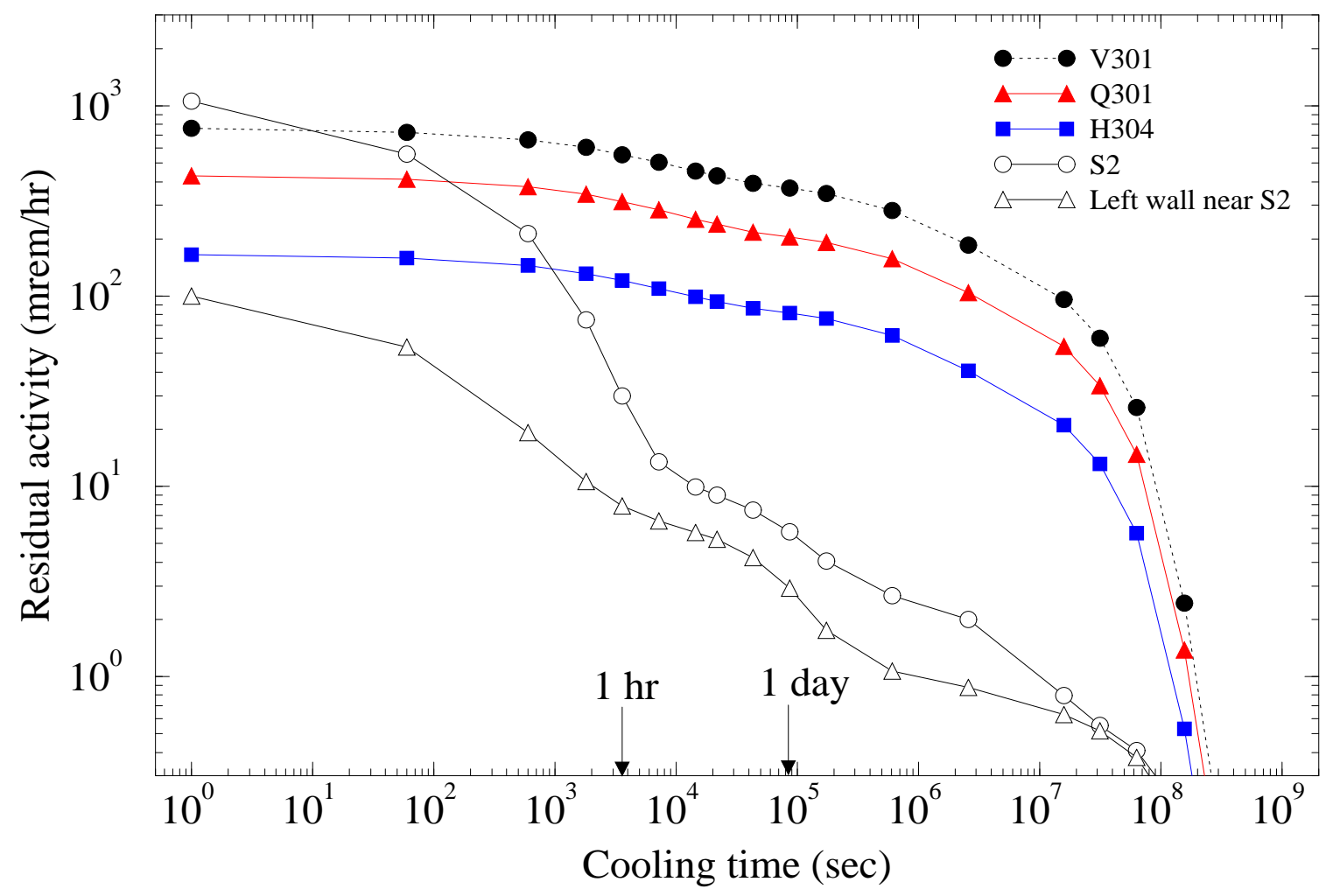

Figure 16: The calculated contact residual dose (mrem/hr) for the aisle side of several hottest elements of the beam line after a continuous 1-year irradiation.

\subsection{Prompt dose rate in beam loss monitors}

In order to provide permanent monitoring of the beam loss at normal operation, a number of beam loss monitors (BLMs) are installed in the collimation region. Near each of the quadrupoles there is a BLM located $60 \mathrm{~cm}$ above the beam line elevation. The predicted prompt dose rate for the monitors can be compared to measured dose rate and, therefore, can serve for verification purposes. The predicted dose rates are given in Table 4.

One can see that the region between Q305 and Q307, where the electron cooling system is installed, is protected against the radiation much better than other parts of the region. In part, this lower radiation from Q305 to Q307 is due to the concrete shield wall upstream of 
Table 4: The calculated prompt dose rate for the BLMs in the MI collimation region.

\begin{tabular}{|c|r|}
\hline Location & Dose rate (rem/hr) \\
\hline Q230 & 1500 \\
Q231 & 130 \\
Q232 & 260 \\
Q301 & 1500 \\
Q302 & 380 \\
Q303 & 2500 \\
Q304 & 340 \\
Q305 & 50 \\
Q306 & 30 \\
Q307 & 1000 \\
Q308 & 400 \\
Q309 & 700 \\
Q310 & 90 \\
\hline
\end{tabular}

Q305 which absorbs stray radiation including the flux of neutrons from upstream collimators.

\section{Conclusions}

The radiation shielding studies were performed for the MI collimation system that comprises a primary horizontal collimator and four secondary ones. The following problems were addressed: (i) surface water activation; (ii) dose load to magnet coils; (iii) distribution of residual activity around the region.

It was shown that after a year of operation the activation of surface water around the hottest spot-secondary collimator S2-is about $32 \%$ of total limit. At the same time, activation of groundwater (aquifer) is negligible - much less than $1 \%$ of total limit.

The highest dose - up to 1 MGy per year-is observed in coils of the Q301 quadrupole which is upstream of the secondary collimator S1. For the magnet this means the lifetime of about four years. For all the other bending dipoles, trim dipoles, and quadrupoles the absorbed dose in the hottest spot varies from 0.1 up to $0.5 \mathrm{MGy} / \mathrm{yr}$ which means the lifetime of about eight years and more.

The predicted residual activity around the region reveals several small hot spots between Q230 and Q302. The issue can be resolved by means of a local shielding. Marble layers are used to cover the external surface of the secondary collimators and reduce the residual dose down to an acceptable level. The advantage of using marble is in its extremely low residual activation while providing significant absorption of $\mathrm{MeV}$ gammas generated in the steel core of the collimators. 


\section{Acknowledgements}

The author is grateful to Bruce Brown, Alexandr Drozhdin, David Johnson, Ioanis Kourbanis, Nikolai Mokhov, and Vladimir Sidorov of Fermilab for help and fruitful discussions.

\section{References}

[1] “The Proton Plan,” Beams-doc-1441-v1 (2004).

[2] A.I. Drozhdin, B.C. Brown, D.E. Johnson, K. Koba, I. Kourbanis, N.V. Mokhov, I.L. Rakhno, V.I. Sidorov, “Collimation System Design for Beam Loss Localization with Slipstacking Injection in the Fermilab Main Injector," Fermilab-Conf-07-249-AD (2007).

[3] M.A. Kostin, O.E. Krivosheev, N.V. Mokhov, I.S. Tropin, "An Improved MAD-MARS Beam Line Builder: User's Guide,” Fermilab-FN-0738-rev (2004).

[4] N.V. Mokhov, Fermilab-FN-628 (1995); N.V. Mokhov et al., Rad. Prot. Dosimetry, v.116, p.99 (2005); N.V. Mokhov et al., Int. Conf. on Nucl. Data Sci. Technology, AIP Conf. Proc., v.769, p.1618 (2005); N.V. Mokhov, S.I. Striganov, "MARS15 Overview," Hadronic Shower Simulation Workshop, AIP Conf. Proc., v.896, p.50 (2006); http://www-ap.fnal.gov/MARS/.

[5] B.C. Brown, N.V. Mokhov, Private communication, Fermilab (2007).

[6] J.-F. Ostiguy, Private communication, Fermilab (2007).

[7] N.V. Mokhov and B.C. Brown, "MARS15 Calculations for MI8 Collimator Design," Fermilab-TM-2359-AD (2006).

[8] “Fermilab Radiological Control Manual," http://www-esh.fnal.gov/FRCM/.

[9] J.D. Cossairt, "Use of a concentration-based model for calculating the radioactivation of soil and groundwater at Fermilab," Fermilab Note EP8; J.D. Cossairt, A.J. Elwyn, P. Kesich, A. Malensek, N. Mokhov, and A. Wehmann, "The concentration model revisited," Fermilab Note EP-17; http://www-esh.fnal.gov/pls/default/esh_home_page.page?this_page=11013/.

[10] I. Kourbanis, Private communication, Fermilab (2007).

[11] O.E. Krivosheev, N.V. Mokhov, “The Proton Driver Design Study," Chapter 10, Fermilab, TM-2136 (2000).

[12] B.C. Brown, "Main Injector Collimation System Hardware," Beams-doc-2881 (2007). 